\title{
Cerebellum regulates the timing of periaqueductal grey neural encoding of fear memory and
} the expression of fear conditioned behaviours.

Authors: *Lawrenson $\mathrm{CL}^{1}$, * Paci $\mathrm{E}^{1}$, Drake $\mathrm{RAR}^{1}$, Lumb BM${ }^{1}, \mathrm{Apps} \mathrm{R}^{1}$

Affiliation: ${ }^{1}$ School of Physiology, Pharmacology \& Neuroscience, University of Bristol, UK

Contributions: *Joint first author.

\section{Abstract:}

The pivotal role of the periaqueductal grey (PAG) in fear learning is reinforced by the identification of neurons in rat ventral (VPAG) that encode fear memory through signalling the onset and offset of an auditory conditioned stimulus during retrieval. Within this framework, understanding of cerebellar contributions to survival circuits is advanced by the discovery that: (i) reversible inactivation of the medial cerebellar nucleus (MCN) during fear consolidation (a) reduces the temporal precision of vPAG offset, but not onset responses and (b) increases rearing behaviour during retrieval, and (ii) chemogenetic inhibition of the MCN-vPAG projection during fear acquisition (a) reduces the emission of fear-related ultrasonic vocalisations and (b) slows the extinction rate of fear-related freezing. These findings show that the cerebellum regulates fear memory processes at multiple timescales and in multiple ways. The current findings indicate that dysfunctional interactions in the cerebellar-survival network may underlie fear-related disorders and comorbidities.

\section{Impact Statement:}

Cerebellar-periaqueductal grey interactions contribute to fear conditioned processes and, as such, provide a novel target for treating psychological conditions including post-traumatic stress disorder. 


\section{Introduction}

The periaqueductal grey (PAG) lies at the hub of central networks that co-ordinate survival, including coping behaviours; from those subserving homeostatic and reproductive functions to those mediating defensive, fear-evoked coping responses. Neurons in the functionally distinct longitudinal columns of the PAG co-ordinate different aspects of survival behaviours. Of particular relevance to the current study, fear-evoked freezing is mediated by neurons in its ventral sector (vPAG; Vianna et al., 2001; Walker and Carrive, 2003; Tovote et al., 2016; Watson et al., 2016). Fear-related behaviours coordinated by the PAG also extend to the expression of $22 \mathrm{kHz}$ ultrasonic vocalizations (USVs; Kim et al., 2013; Ouda et al., 2016) and risk assessment activity such as rearing (Sandner et al., 1987; Clelland et al., 2010). Furthermore, at a cellular level, electrophysiological studies have found that neurons in VPAG encode associatively conditioned fear memory (Watson et al., 2016; Wright et al., 2019).

Central nervous system survival networks involving the PAG are well documented (Tovote et al., 2015). Until recently, the cerebellum was generally considered not to be part of this network. However, there is good evidence in cats and rodents that vermal regions of the cerebellum contribute to motor and autonomic components of defensive states; inactivation of vermal cerebellar cortex (lobules IV-VIII), or one of its main output nuclei (medial cerebellar nucleus, MCN, aka fastigial nucleus), leads to deficits in fear-related behaviours such as context conditioned bradycardia (Supple et al., 1990); the expression of innate fear (Supple et al., 1987; Koutsikou et al., 2014); and also fear conditioned freezing behaviour (Asdourian et al., 1970; Albert et al., 1985; Sacchetti et al., 2005; Koutsikou et al., 2014). In addition, the emission of USVs has been related to cerebellar function (Fujita et al., 2008; Fujita-Jimbo et al., 2014; Toledo et al., 2019), and inactivation of the cerebellar vermis during innate and conditioned fear unmasks risk assessment rearing behaviour (Koutsikou et al., 2014).

The cerebellum is reciprocally connected to many brain regions associated with survival networks (Sacchetti et al., 2009; Strick et al., 2009; Apps \& Strata, 2015) and in all mammalian species studied so far (cat, rabbit, rat, mouse, human), this includes interconnections with the vPAG (Whiteside et al., 1953; Teune et al., 2000; Nisimaru et al., 2013; Koutsikou et al., 2014; Cacciola et al., 2019; Vaaga et al., 2020; Frontera et al., 2020). Thus, the dependence of survival behaviours on the integrity of the cerebellum, together with reciprocal connections between the vPAG and the cerebellum raise the possibility of an important role of cerebellar interactions with the PAG in the expression of such behaviours.

In particular, an emerging concept is that the cerebellar vermis and its output nucleus MCN are involved in the control of an integrated array of fear-related functions, including fear memory and 
fear-induced behaviours such as freezing. Important insights into the role of the murine MCN-vPAG pathway in modulating VPAG fear learning and memory have been provided recently (Vaaga et al., 2020; Frontera et al., 2020). However, it is not known whether PAG neural encoding of fear memory is dependent on the integrity of its cerebellar input. More generally, given the well-established role of the cerebellum in the co-ordination of movements and in particular, the representation of temporal relationships (e.g. Xu et al., 2006; Koch et al., 2007; Bares et al., 2007; Spencer \& Ivry, 2013; Johansson et al., 2016) it remains to be determined whether the cerebellum enables the survival circuit network to elicit behaviourally appropriate responses at different times during fear conditioning. Effects during retrieval and extinction of a fear conditioned response are of particular interest because deficits in extinction processes are thought to underly psychological conditions such as post-traumatic stress disorder (Bremner et al., 1999; Herry et al., 2010; Milad et al., 2012) and have also been related to chronic pain phenotype (Ji et al., 2018).

The experiments reported here used a combination of electrophysiological, behavioural and interventionist approaches in an auditory cued fear conditioning paradigm in rats to interrogate the role of MCN interactions with the VPAG in the expression of fear-related behaviours. Our main findings are that: (i) VPAG encodes temporally precise information about the onset and offset of a fear conditioned stimulus and that these two neural signals may be generated by independent mechanisms; (ii) MCN output regulates the temporal accuracy of VPAG encoding of fear conditioned stimulus offset but not onset during retrieval (early extinction) of the conditioned response; (iii) MCN output also regulates multiple aspects of survival behaviour at different times during fear conditioning: during acquisition the emission of USVs and the suppression of rearing and also the rate of extinction of conditioned freezing during retrieval. In summary, the present study provides evidence that the cerebellum regulates the ability of the PAG to encode a fear memory trace with temporal precision and also regulates the time of occurrence of appropriate patterns of behaviour during fear acquisition and retrieval.

\section{Results}

VPAG unit responses during an auditory cued fear conditioning paradigm

As a first step, an auditory cued fear conditioning paradigm (Fig. 1A) was used to investigate how single unit activity in the vPAG ( $\mathrm{n}=11$ animals) responded to fear acquisition and subsequent retrieval and extinction of fear conditioned responses (Fig. 1B-E). In seven of these animals a dual microdrive included tetrodes that were also implanted in the contralateral MCN to record local field potential 
1 activity. The remaining four animals had a single microdrive for VPAG recording combined with

2 bilateral cannulae implanted to target $\mathrm{MCN}$. In all animals the position of tetrodes (and where

3 appropriate cannulae) was histologically verified. The majority of PAG tetrode recording sites were

4 located in the VPAG, and cannulae or tetrodes implanted in the cerebellum were located close to or

5 within MCN (Supplementary Fig. 1A, B and C). Since similar electrophysiological data were recorded

6 from the PAG obtained from animals with a dual microdrive (PAG and MCN) and those with a single

7 microdrive (PAG, that received a saline infusion into $M C N$ ), the results have been pooled in the analysis

8 below (termed 'control', Figs 2 and 3). There was no significant difference between baseline firing

9 rates across habituation, acquisition, and extinction training (Supplementary Fig. 2, p=0.649, one-way

10 ANOVA). Thus, it seems reasonable to assume that single unit recording was stable over time and

11 comparable between groups. 


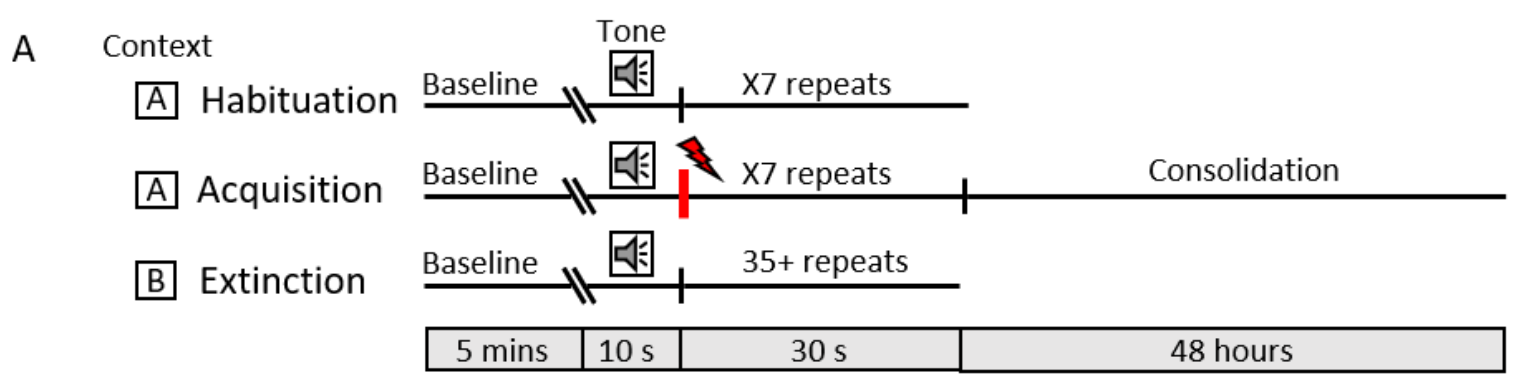

B

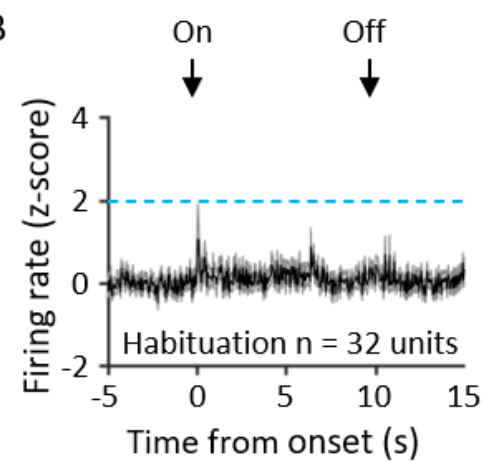

C

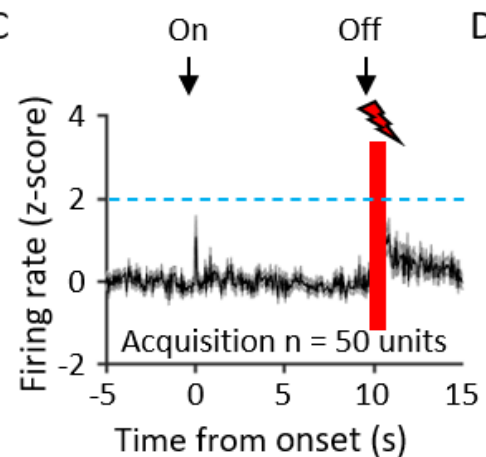

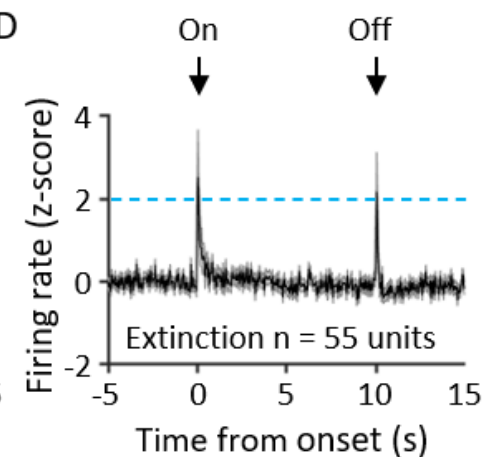

Time from onset $(s)$
$E$

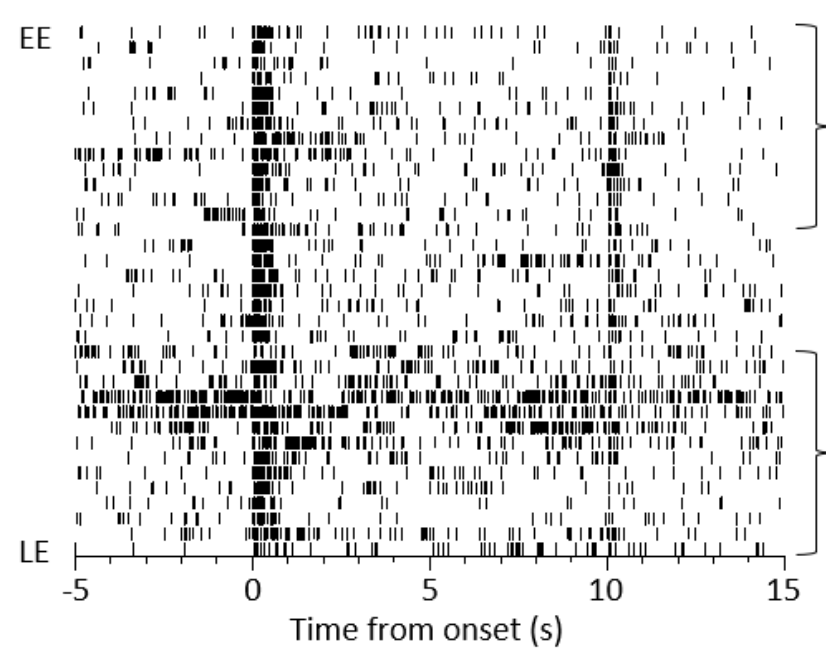

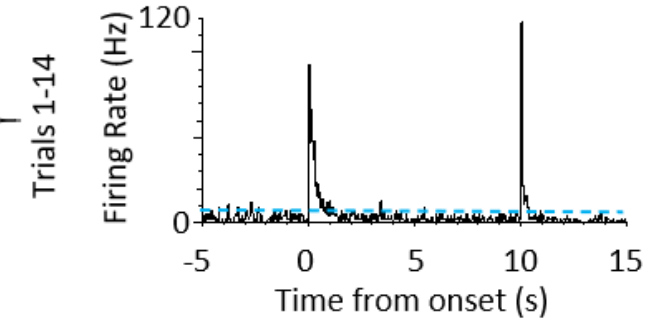

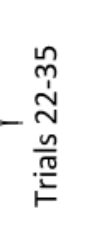

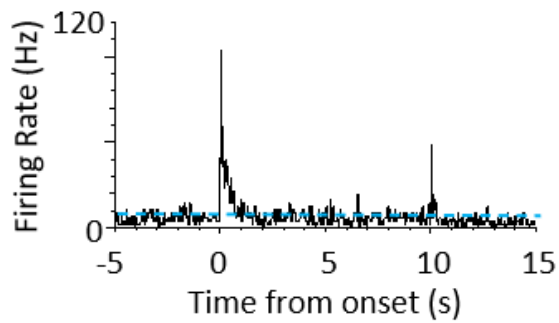

Figure 1. Single unit vPAG responses during auditory cued fear delay conditioning. A) Schematic representation of the fear conditioning paradigm composed of habituation, acquisition and extinction sessions. Habituation and acquisition were carried out in context $A$, whilst extinction training was in context B. During acquisition, a CS tone was paired with a US footshock (see Methods for details). In cannula implanted animals, muscimol or saline was injected into the MCN following acquisition during the consolidation phase before retrieval of the CS+ during extinction training. B) Peri event time histogram (PETH) showing average firing rate of all available single units $(n=32)$ recorded during presentation of the unconditioned tone (10 s) during habituation in control animals. C) Same as $B$ but all available single units $(n=50)$ recorded during acquisition (shaded red and lightening symbol indicate time of US footshock). D) Same as $B$ but all available single units $(n=55)$ recorded during presentation of the unreinforced CS+ during extinction. For B-D, individual unit activity was z-score normalised to a 5 second baseline before tone onset. PETHs show mean \pm SEM; 40ms bins. E) Example type 1 onset and offset single unit recorded during extinction training. Data displayed as raster plot from early extinction (EE) to late extinction (LE) with corresponding PETH for EE (trials 1-14) and LE (trials 22-35) (right; 40 ms bins, time zero onset of CS+. The dotted blue horizontal line represents significance level $(p<0.05)$. 
A total of 32 vPAG units were recorded during habituation (Fig. 1 B). The large majority ( $75 \%, n=24 / 32$ ) were unresponsive to the unconditioned auditory tone; the remainder responded (see Methods for definition) to tone onset (either increasing, $n=6 / 32$ units or decreasing firing rate, $n=2 / 32$ units); and in some of these cases (15.6\%) also to tone offset (either increasing $3 / 32$ units or decreasing firing rate $2 / 32$ ).

We recorded the activity of 50 VPAG units during acquisition where the CS tone was paired with the US footshock at tone offset (Fig. 1C). A small proportion of units (18.5\%) responded to CS onset. Following the US, some units (20\%) also displayed an increase in firing rate which presumably reflects sensory afferent drive to the PAG as a result of the aversive peripheral stimulus (e.g. (Sanders et al., 1980; Heinricher et al., 1987; Sharma et al., 1999).

During retrieval and extinction of the conditioned response we recorded a total sample of 55 VPAG units in control animals. In retrieval during early extinction (EE) 29 units (52.7\%) displayed a transient increase in activity during presentation of the unreinforced conditioned stimulus (CS+, Fig. 1D, E). In keeping with a previous classification of PAG unit activity during extinction training (Watson et al., 2016) these units are therefore defined as type 1 . The pattern of response was typically a phasic increase in activity at CS+ onset but an additional feature not previously reported for VPAG units was also a phasic increase in activity at CS+ offset (Fig. 1D, E). A total of 21/29 (72.4\%) type 1 units responded to both $\mathrm{CS}+$ onset and CS+ offset, but a small proportion only responded to CS+ onset $(10.3 \%, n=3 / 29)$ or only to $C S+$ offset $(17.3 \%, n=5 / 29)$. This raises the possibility that $C S+$ onset and offset responses may be partly independent of one another. We have therefore termed them type 1 onset and type 1 offset responses, respectively.

Of the remainder of the sample of VPAG units recorded in control animals during $E E, 22(40 \%)$ demonstrated no significant change in firing rate and were therefore classed as type 2 (Watson et al., 2016). Four units (7\%) responded to CS+ onset with a decrease in firing rate ( 2 of these units also reduced their firing rate at $\mathrm{CS}+$ offset) and therefore were classed as type 4 . No type 3 units (biphasic response) were observed.

Since the large majority of responsive VPAG units recorded in control experiments were type 1 the following analysis is confined to consideration of their activity during fear conditioned retrieval and extinction. The majority of type 1 onset units (75\%, 18/24 units) and type 1 offset units (73\%, 19/26 units) showed significant decreases in responsiveness during extinction training, as measured by the integrated area of response during CS+ trials in EE versus late extinction (LE, Fig. 2A,C control, type 1 onset units: $p<0.0001$, paired t test; type 1 offset units, Fig $3 A, C, p=0.0003$, paired t test). For type 1 onset responses this reduction was not evident for mean peak z-score (Fig. 2D, decrease of 13.8\% 
bioRxiv preprint doi: https://doi.org/10.1101/2021.02.17.431584; this version posted February 17, 2021. The copyright holder for this preprint (which was not certified by peer review) is the author/funder, who has granted bioRxiv a license to display the preprint in perpetuity. It is made available under aCC-BY 4.0 International license.

1 from EE to LE; paired t test; $p=0.197$ ) but was evident for type 1 offset responses (Fig. 3D, decrease of $2 \quad 67.0 \%$ from EE to LE; paired t test; $p=0.011$ ). This distinction between onset and offset responses 3 provides additional evidence to suggest they may be evoked by separate mechanisms. 
A
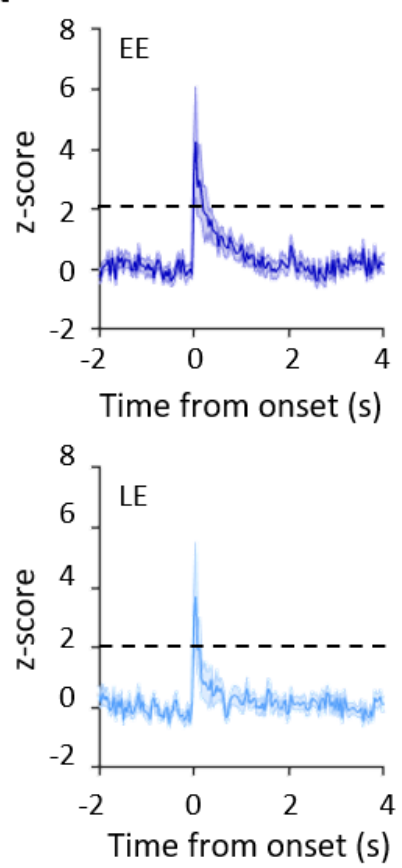

B
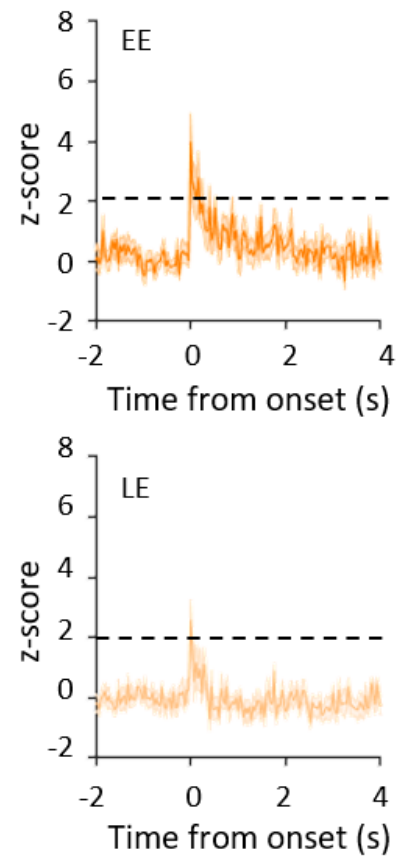

C

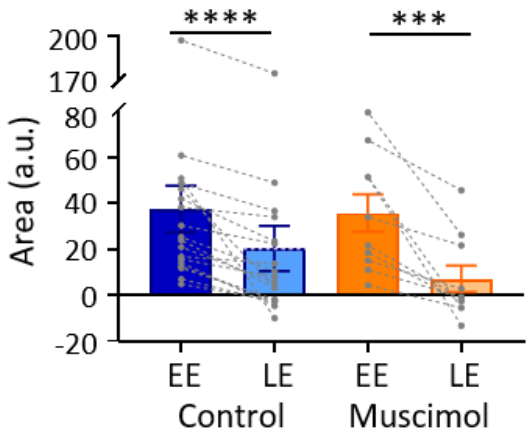

D

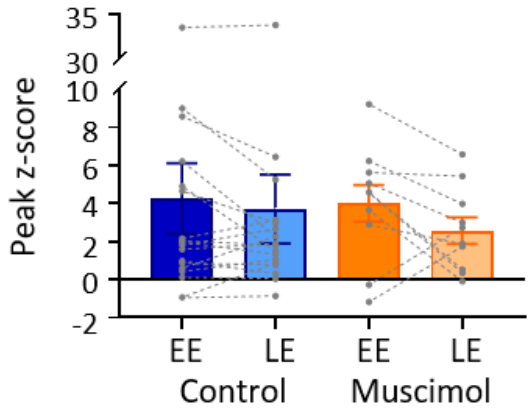

$\mathbf{F}$

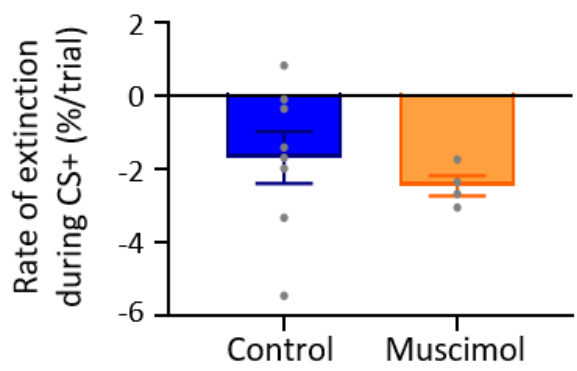

H

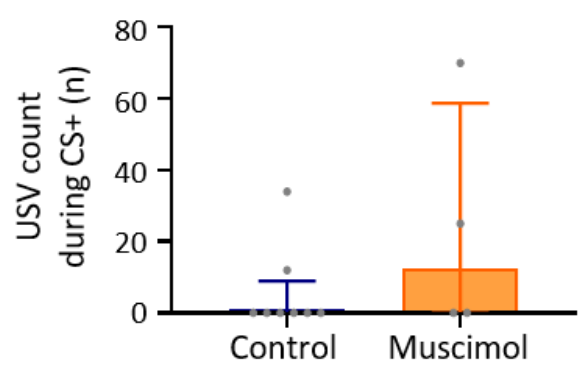

1
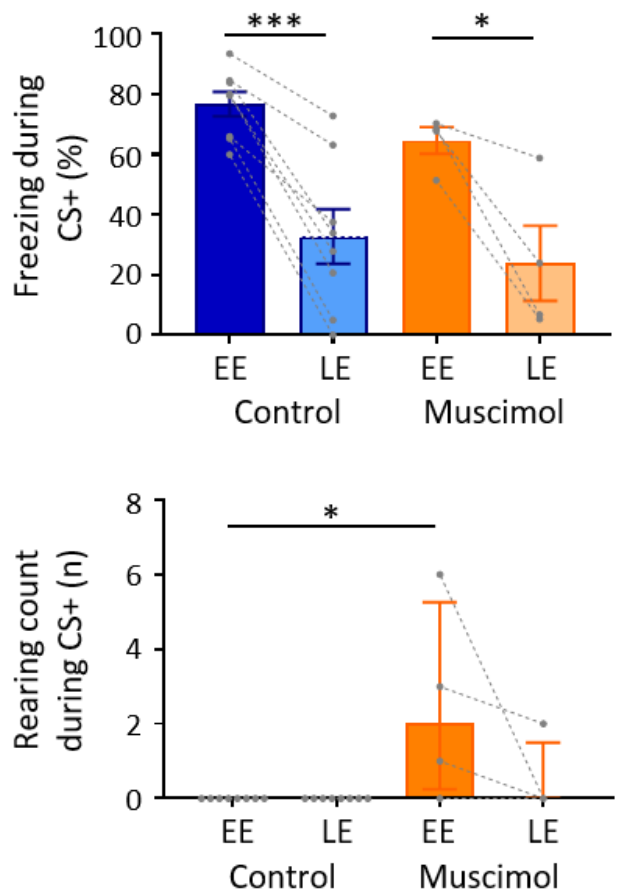

Figure 2. Effect of MCN inactivation during consolidation on vPAG type 1 onset responses and fear behaviours during extinction. A) Group data for control animals showing average $z$-scored type 1 onset responses ( $n=18$ units) during early extinction (EE, upper panel) and late extinction (LE, lower panel); solid lines in each plot show mean z-score, shaded regions \pm SEM; horizontal dashed lines show +2SD from baseline. B) Same as A but grouped data for muscimol animals ( $n=10$ single units). C) Bar charts showing average type 1 onset response area (arbitrary units) during EE and LE for single units recorded in control versus muscimol animals. Individual data points connected with dashed lines show change in mean response area for each single unit over extinction training. Bars show group means \pm SEM. ${ }^{* * *} p<0.0001, * * * p<0.001$. D) Same as $C$ but grouped data for peak z-score. E) The percentage of total time the CS+ was presented that animals displayed freezing behaviour during EE or LE in control ( $n=8$ rats) versus muscimol ( $n=4$ rats) experiments. Individual data points connected with 
bioRxiv preprint doi: https://doi.org/10.1101/2021.02.17.431584; this version posted February 17, 2021. The copyright holder for this preprint (which was not certified by peer review) is the author/funder, who has granted bioRxiv a license to display the preprint in perpetuity. It is made available under aCC-BY 4.0 International license.

dashed lines show change in mean freezing time per animal over extinction training. Bars show group means \pm SEM. ${ }^{* * *} \mathrm{p}<0.001, * \mathrm{p}<0.05$. $\mathrm{F}$ Rate of extinction as measured by the change in freezing percentage during presentation of the CS+ over the first $21 \mathrm{CS}+$ presentations during extinction training in control ( $n=8$ rats) versus muscimol ( $n=4$ rats) experiments. Individual data points show mean rate of change per animal. Bars show group means \pm SEM. G) The total number of rears during presentation of the CS+ in extinction training in control $(n=8$ rats) versus muscimol ( $n=4$ rats) experiments. Individual data points connected with dashed lines show change in mean number of rears per animal over extinction training. Bars show group median and IQR. (Mann Whitney test, ${ }^{*}$ p < 0.05). H) Total number of ultrasonic vocalisations (USVs) during presentation of the CS+ in extinction training in control ( $n=8$ rats) versus muscimol ( $n=4$ rats) experiments. Individual data points show mean count per animal. Bars show group median and IQR. 
A
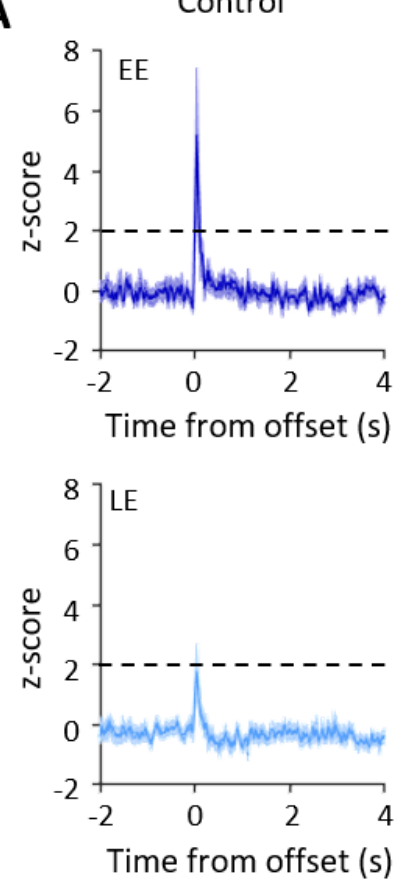

B
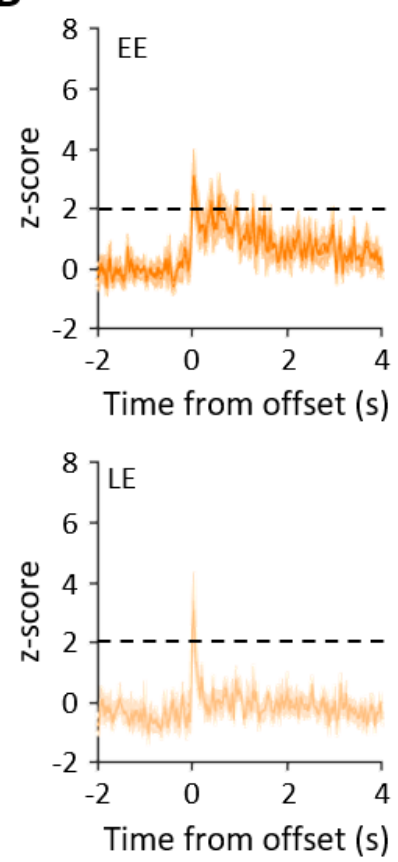

C

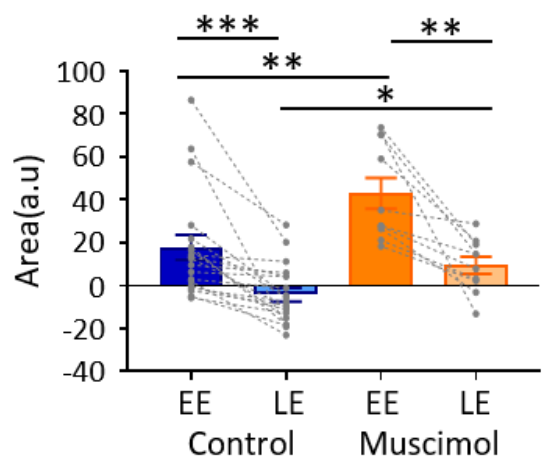

D

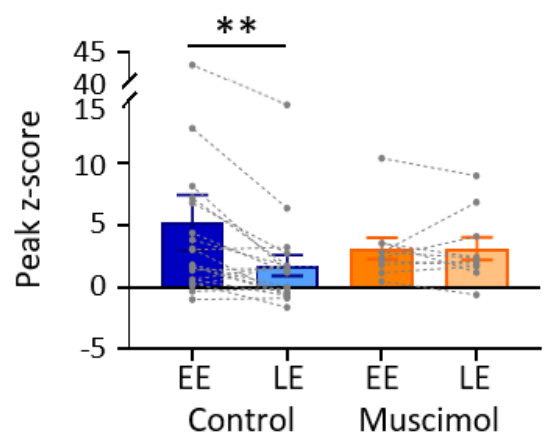

$\mathbf{F}$

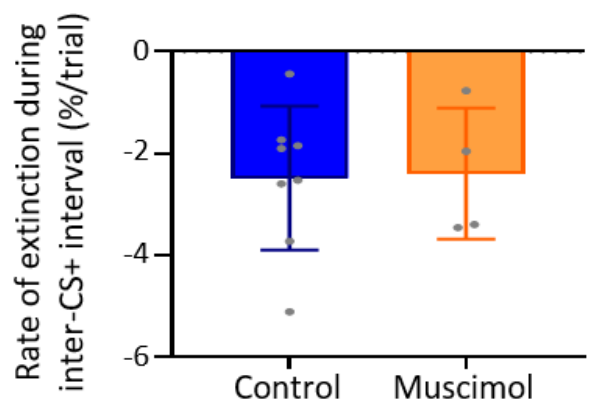

H

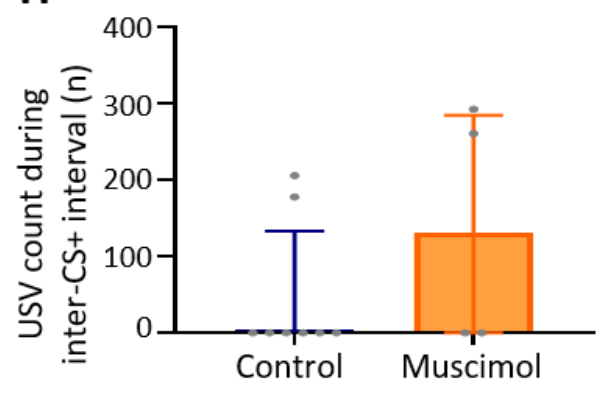

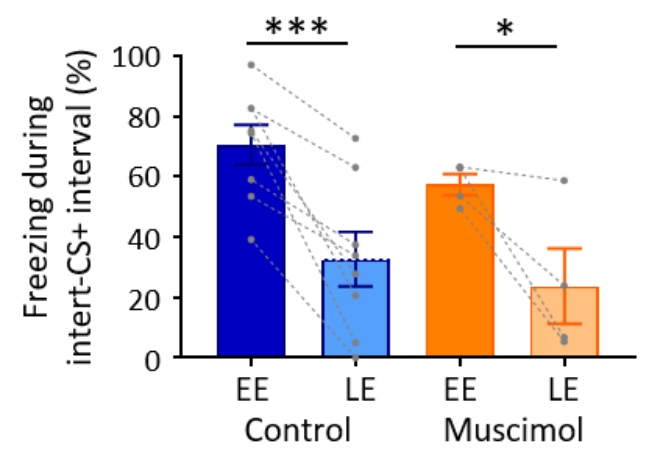

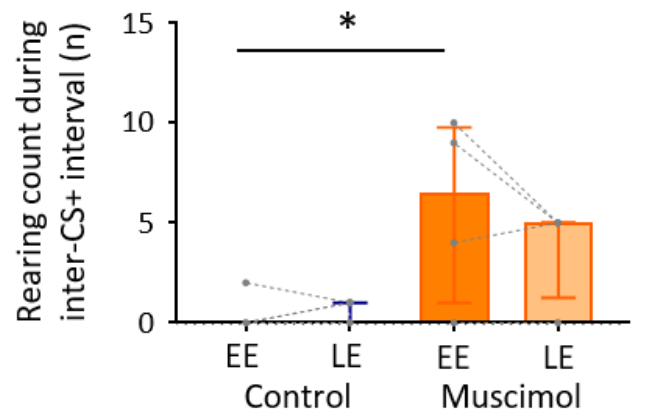

Figure 3. Effect of MCN inactivation during consolidation on vPAG type 1 offset responses and fear behaviours during extinction. A) Group data for control animals showing average z-scored type 1 offset responses ( $n=19$ single units) during early extinction (EE, upper panel) and late extinction (LE, lower panel); solid lines in each plot show mean z-score, shaded regions $\pm S E M$; horizontal dashed lines show +2SD from baseline. B) Same as A but grouped data for muscimol animals ( $n=10$ single units). C) Bar charts showing average type 1 offset response area (arbitrary units) during EE and LE for single units recorded in control versus muscimol animals. Individual data points connected with dashed lines show change in mean response area for each single unit over extinction training. Bars show group means \pm SEM. Paired t test, $* * * p<0.001, * * p<0.01$; Unpaired t test, ${ }^{* *} p<0.01$, $*_{p}<0.05$. D) Same as $C$ but grouped data for peak $z$-score. Paired t test, $* * p<0.01$. E) The percentage of total time during the inter-CS+ interval that animals displayed freezing behaviour during EE or LE in control ( $n=8$ rats) 
versus muscimol ( $n=4$ rats) experiments. Individual data points connected with dashed lines show change in mean freezing time per animal over extinction training. Bars show group means \pm SEM. Paired t test, $* * *$ $p<0.001, * p<0.05$. F) Rate of extinction as measured by the change in freezing percentage during the inter-CS+ interval for the first $21 \mathrm{CS}+$ presentations during extinction training in control $(n=8$ rats) versus muscimol $(n=4$ rats) experiments. Individual data points show mean rate of change per animal. Bars show group means \pm SEM. G) The total number of rears in extinction training during the inter-CS+ interval in control ( $n=8$ rats) versus muscimol ( $n=4$ rats) experiments. Individual data points connected with dashed lines show change in mean number of rears per animal over extinction training. Bars show group median and IQR (Mann Whitney test, ${ }^{*} p<0.05$ ). H) Total number of ultrasonic vocalisations (USVs) during extinction training in the inter-CS+ interval for control ( $n=8$ rats) versus muscimol ( $n=4$ rats) experiments. Individual data points show mean count per animal. Bars show group median and IQR.

\section{vPAG offset responses during trace conditioning.}

For the results described above a delay classical conditioning paradigm was used during acquisition in which the unconditioned stimulus (US) was timed to occur at CS offset (Fig. 1A, see Methods). During $\mathrm{EE}$ (retrieval) an increase in unit activity at CS+ offset may therefore signal the end of the conditioned tone and/or timing of the expected US. To examine this, in one animal, a 1 second trace delay was introduced between the CS and US during acquisition trials. Four VPAG units were recorded during extinction training and all responded at CS+ offset and did not respond at the time when the US would have been expected to occur $1 \mathrm{sec}$ later (Supplementary Fig. 3). Although caution is clearly needed when interpreting a small sample, our results are consistent with vPAG activity at CS+ offset signalling the end of the conditioned stimulus rather than the time of the expected occurrence of the unconditioned stimulus.

\section{The effect of temporary MCN inactivation during fear consolidation on VPAG activity}

In 4 additional animals, muscimol was infused into the MCN to reversibly block cerebellar output during consolidation of the fear associative memory prior to extinction training (termed 'muscimol' in extinction sessions, Figs 2 and 3). Effects of the muscimol infusion on general motor co-ordination were carefully monitored. Immediately after the infusion all animals $(n=4)$ displayed ataxia, providing a positive control that the muscimol was disrupting cerebellar activity. The severity of the ataxia gradually reduced over several hours and the animals were behaviourally normal after $24 \mathrm{hrs}$. After a total delay of $48 \mathrm{hrs}$, to ensure complete washout of the drug, the animals were exposed to the unreinforced CS+ in extinction training. There was no significant difference between baseline firing rates across habituation, acquisition, and extinction training (Supplementary Fig. 2, p=0.079, one-way ANOVA). A total of 14 type 1 vPAG units were recorded during extinction. Similar to the control results described above, the majority (71\%, 10/14 units) of type 1 onset units (Fig. 2B) showed a reduction in responsiveness during extinction training as measured by mean change in integrated area of response 
1 (Fig. 2C, on average the integrated area of response was $77.8 \%$ smaller between $\mathrm{EE}$ and $\mathrm{LE}, \mathrm{p}=0.002$,

2

3 paired $t$ test). In keeping with the findings from control animals, no significant difference was found for mean peak z-score (Fig. 2D, peak $0.7 \%$ smaller between EE and LE, $p=0.971$, paired t test). There was also no statistically significant difference between control and muscimol animals for CS+ onset responses in $\mathrm{EE}$ or LE for either measure of response (Fig. $2 \mathrm{C}$ and $\mathrm{D}$, mean integrated area in $\mathrm{EE}, \mathrm{p}=$ 0.689 , unpaired $t$ test; mean integrated area in $\mathrm{LE}, \mathrm{p}=0.351$, unpaired $t$ test; mean peak z-score in $\mathrm{EE}$, $p=0.919$, unpaired $t$ test; mean peak $z$-score in $L E, p=0.654$, unpaired $t$ test,).

By marked contrast, inspection of Figure $3 A, B$ shows that by comparison to controls ( $n=23$ units), there was a clear difference during EE in the pattern of response of VPAG units to CS+ offset in muscimol experiments ( $n=10$ units). On average, the integrated area of response in muscimol experiments increased by $58.5 \%$ by comparison to control (Fig. 3C, $p=0.002$, unpaired $t$ test). Inspection of Figure $3 A, B$ shows there was also a reduction in mean peak $z$-score (muscimol mean peak was $60 \%$ of control) but this was not statistically significant (Fig. 3D, $p=0.515$, unpaired $t$ test). In LE, the integrated area of response was also significantly different. On average, muscimol experiments had a significantly increased area of response (41.6\% larger) than control animals (Fig 3C, $p=0.014$, unpaired t test). Similar to EE, the mean peak z-score in LE was also not significantly different between muscimol and control experiments (Fig 3D, $p=0.32$, unpaired $t$ test). Taken all together these data therefore suggest that during extinction training the temporally precise pattern of response of VPAG units at CS+ onset is mainly unaffected by disruption of MCN during consolidation, but the activity at CS+ offset is disrupted during $\mathrm{EE}$, providing additional evidence that the two responses are independent of one another.

The effect of temporary MCN inactivation during fear consolidation on behaviour

\section{(i) Effects during presentation of the CS+ in extinction training}

To investigate whether the muscimol infusion into $\mathrm{MCN}$ during consolidation had an effect on subsequent expression of fear-conditioned behaviour, several defence-related responses were measured during presentation of the unreinforced CS+: freezing (\% during extinction and extinction rate), rearing activity and number of fear-related ultrasonic vocalisations (USVS). Both control and muscimol animals showed extinction of the conditioned freezing response between EE vs LE (Fig. 2E. Control, EE freezing $=76.7 \pm 4.1 \%$ vs LE freezing $=32.6 \pm 9.04 \%, p=0.0002$; Muscimol, EE freezing= $64.6 \pm 4.4 \%$ vs $L E$ freezing $=23.7 \pm 12.4 \%, p=0.035$, paired $t$ tests). No significant differences were detected between control and muscimol animals in the percentage of time freezing during EE or LE (Fig. 2E. EE, $p=0.098 ; \mathrm{LE}, \mathrm{p}=0.579$, unpaired $t$ test), nor was there a difference detected in rate of extinction of freezing (Fig. 2F. Control, $-1.7 \pm 2.0$; Muscimol, $-2.5 \pm 0.6 ; p=0.473$, unpaired $t$ test). 
However, muscimol animals $(n=4)$ displayed a significant increase in rearing activity during $E E$ compared to controls (Fig. 2G, $n=8$ animals, median 2.0 rears during CS+ by comparison to control of 0 rears, $p=0.018$, Mann Whitney test). This difference was not evident in LE ( $p=0.333$, Mann Whitney test). In muscimol animals, rearing activity reduced in LE, but this was not significantly different from EE $(p=0.250$, Wilcoxon test $)$.

In terms of ultrasonic vocalisations (USVs) 2/8 (25\%) of control animals and 2/4 (50\%) of muscimol animals emitted USVs during presentation of the CS+. In terms of the mean total number of USVs per animal in control versus muscimol groups across all extinction training there was no significant difference (Fig. 2H, p = 0.321, Mann Whitney test).

In summary, for the various behavioural measures studied there was no detectable difference between control and muscimol animals during extinction training with the exception of rearing, where in EE (retrieval), MCN inactivation resulted in a significant increase during presentation of the CS+.

\section{(ii) Effects after presentation of the CS+ in extinction training}

To investigate whether MCN inactivation during consolidation has effects on fear behaviour after CS+ offset, the same behavioural measures studied during delivery of the CS+ were quantified during the inter-CS+ interval. In terms of freezing behaviour during the inter-CS+ interval, both control and muscimol animals showed extinction learning similar to that found during presentation of the CS+ (Fig $3 E$, EE vs LE: control, $p=0.0003$, muscimol, $p=0.014$, paired $t$ tests). There were no significant differences in muscimol animals by comparison to controls for either EE or LE (Fig. 3E, EE, p = 0.21. LE, $p=0.929$, unpaired $t$ tests). Nor was there a significant difference in the rate of extinction between groups (Fig. 3F, $p=0.918$, unpaired $t$ test).

In terms of rearing behaviour, during EE muscimol animals displayed significantly more rearing activity during the inter-CS+ interval than control animals (Fig. 3G, median 6.5 rears versus 0 rears during EE, $p=0.024$, Mann Whitney test). During $L E$, although muscimol animals displayed higher numbers of rears (median 5 compared with control animals 0 rears), this difference was not statistically significant (Fig. 3G, $p=0.067$, Mann Whitney test).

The mean total number of USVs across all extinction blocks per animal in muscimol groups was increased during the inter-CS+ interval but this was not significantly different from control (Fig. $3 \mathrm{H}, \mathrm{p}$ $=0.26$, Mann Whitney test). Therefore, based on the available data no systematic differences were detected in the inter CS+ interval between control and muscimol groups in terms of extinction learning or numbers of USVs but a significant difference was detected in terms of an increase in rearing behaviour during EE (retrieval). 
2 To assess changes in neural population activity during extinction, auditory event related potentials 3 (ERPs) were recorded simultaneously from the MCN and vPAG ( $n=5$ animals, Fig. 4). The ERP in the

4

At CS+ onset, the peak-to-peak amplitude of the ERPs recorded in the MCN and VPAG did not show a statistically significant difference during extinction training (Fig. 4A, MCN: EE ERP $=527.1 \pm 169 \mathrm{mV}$ vs LE ERP $=356 \pm 130.5 \mathrm{mV}, \mathrm{p}=0.09$, paired t-test; Fig 4C, vPAG: EE ERP $=539.2 \pm 119.4 \mathrm{mV}$ vs LE ERP $=$ $467 \pm 83.7 \mathrm{mV}, \mathrm{p}=0.466$; paired t-test). By comparison, ERPs recorded at $\mathrm{CS}+$ offset in both the MCN (Fig. 4B) and vPAG (Fig. 4D) showed a significant decrease in amplitude during extinction training in EE versus $L E$ (MCN 23.7\% reduction in ERP size, $E E=575.7 \pm 206.9$ vs $L E=439.1 \pm 165.8, p=0.038$; vPAG 37.2\% reduction in ERP size, $E E=580.4 \pm 133.8$ vs $L E=364.7 \pm 113.2, p=0.002$, paired t-test). Taken together these ERP data therefore suggest that population activity in MCN and VPAG parallel the changes that occur in VPAG single unit peak activity at onset and offset of the CS+ during extinction of a fear conditioned response. 
A CS+ onset MCN CS+ offset

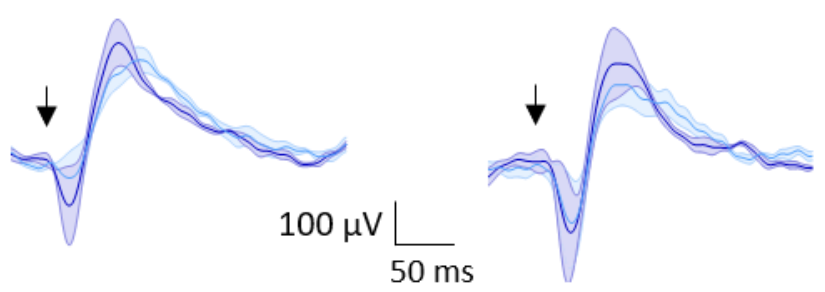

B CS+ onset VPAG CS+ offset
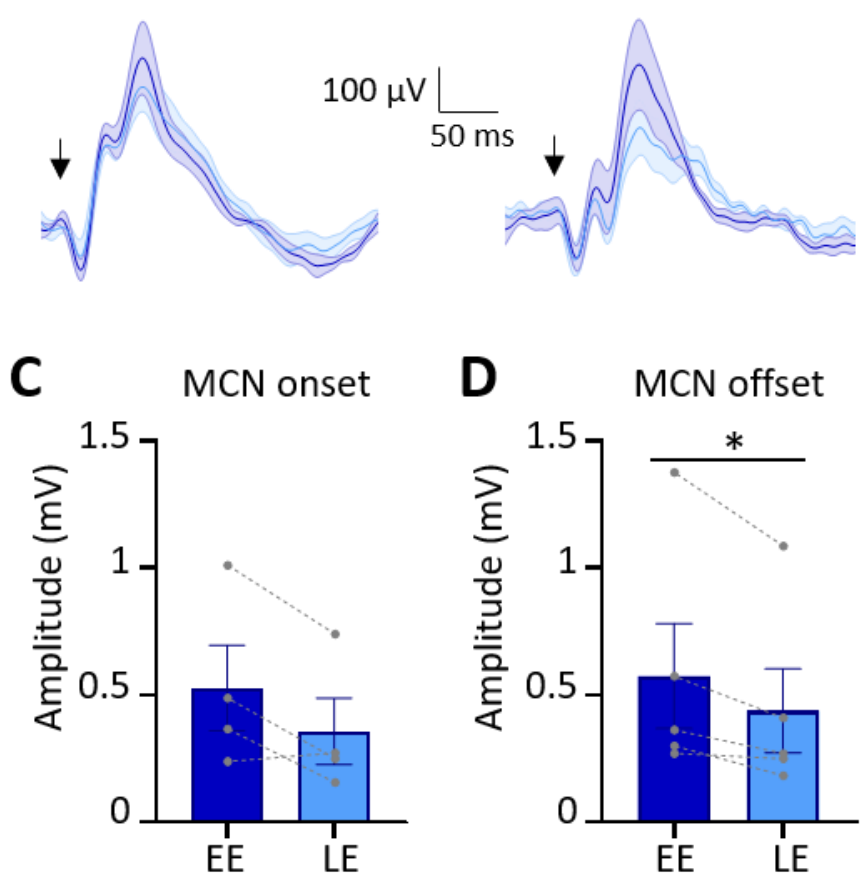

D $\mathrm{MCN}$ offset
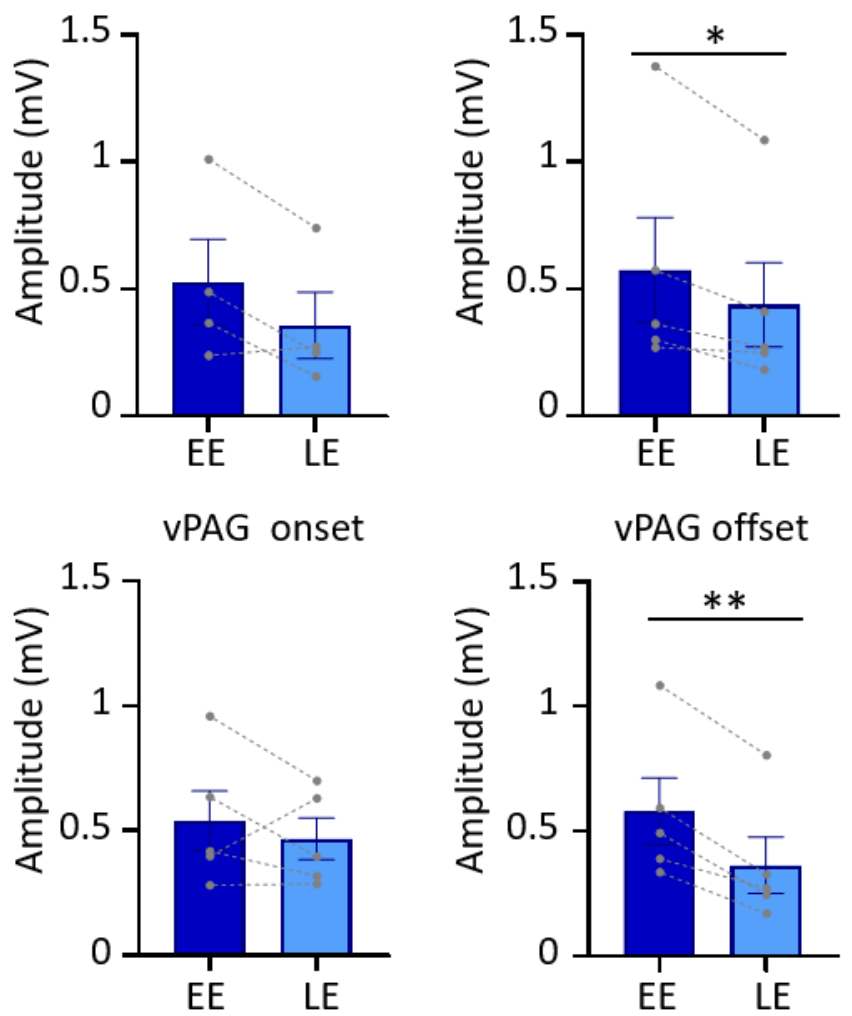

Figure 4. Auditory event related field potentials (ERPs) recorded simultaneously in the MCN and vPAG during extinction. A) Group average ERPs recorded in the MCN in control animals at CS+ onset ( $n=4$ rats) (left hand panel) and CS+ offset ( $n=5$ rats) (right hand panel); arrows indicate time of CS+ onset or offset; each waveform shows mean \pm SEM; dark blue, average ERP during EE, light blue, average ERP during LE. B) Same as A but ERPs recorded simultaneously from the vPAG ( $n=5$ rats). C) Plots showing mean peak to peak amplitude of ERPs recorded at CS+ onset in EE versus LE; upper panel MCN, lower panel vPAG ( $n=4$ and $n=5$ rats respectively, means \pm SEM). Individual data points connected with dashed lines show change in mean amplitude over extinction training. D) Same as $C$ but for $C S+$ offset $\left(n=5\right.$ rats, Paired t-test, $\left.{ }^{*} p<0.05,{ }^{* *} p<0.01\right)$. 
Given that during extinction training: (i) population activity in MCN resembles changes in VPAG population and unit activity; and (ii) the finding that global inactivation of MCN in rats can disrupt encoding in VPAG, it was of interest to determine the extent to which a direct projection exists between the MCN and vPAG. In 7 rats a fluorescently tagged anterograde virus was injected into the MCN (Supplementary Fig. 4). In every case terminal projections in the PAG were primarily localised to its ventrolateral region on the contralateral side.

To investigate the function of this direct MCN-PAG projection, viral vector encoding the inhibitory DREADD hM4D ( $n=10$, AAV-hSyn-hM4D(Gi)-mCherry, termed DREADD, see Methods) or control (pAAV-hSyn-EGFP, $n=9$ ) was injected bilaterally into the MCN. Terminal projections containing the virus were targeted in the VPAG by stereotaxic delivery of CNO (for histology see supplementary Fig $5 A)$.

To determine the effect of MCN-PAG pathway inactivation on conditioned fear behaviours, CNO was infused 15 minutes before acquisition training to inhibit MCN-PAG projections during acquisition and early consolidation. During acquisition there was no significant difference in the percentage of freezing between control versus DREADD animals during CS-US paired presentations (Fig. 5A upper panel, two-way repeated measures ANOVA; Time, $F(4.45,75.64)=16.63, p<0.0001 ;$ Virus, $F(1,17)=$ $0.0889, p=0.769$ ) nor during the inter-CS-US interval (Fig. 5A lower panel, two-way repeated measures ANOVA; Time, $F(3.834,65.19)=17.82, p<0.0001$; Virus, $F(1,17)=2.339, p=0.144)$. However, comparison of the mean total number of USVs per animal in DREADD versus control groups across all acquisition trials showed that during the inter CS-US interval, there was a significant reduction in USVs (Fig. 5B lower panel, a decrease of $36 \%, p=0.032$, Mann Whitney test). This difference was not apparent during CS-US presentations (Fig. 5B upper panel, $p=0.310$, Mann Whitney test). 
A
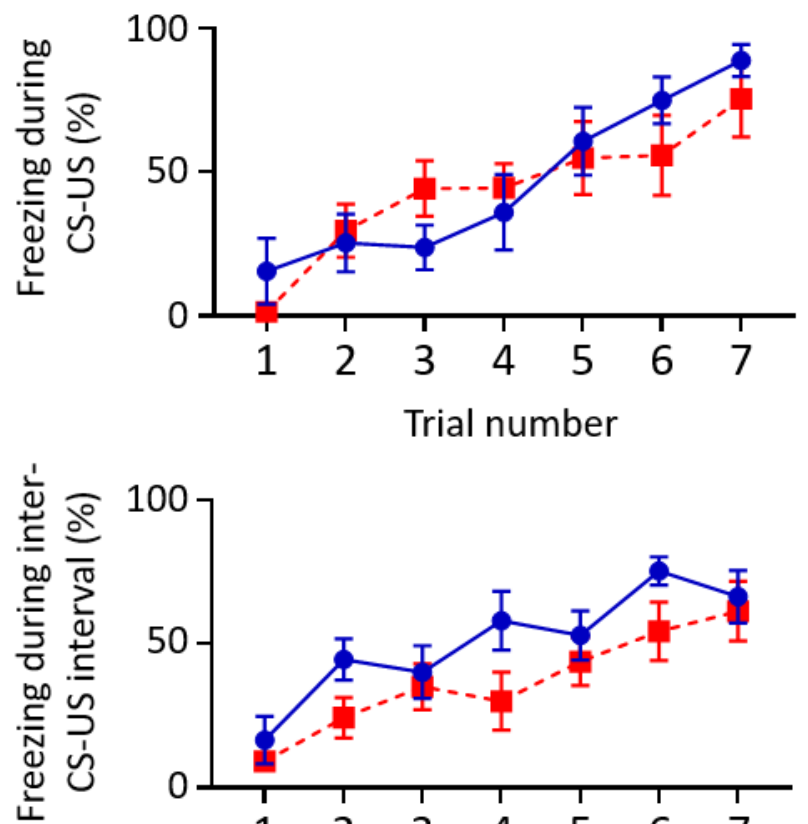

B
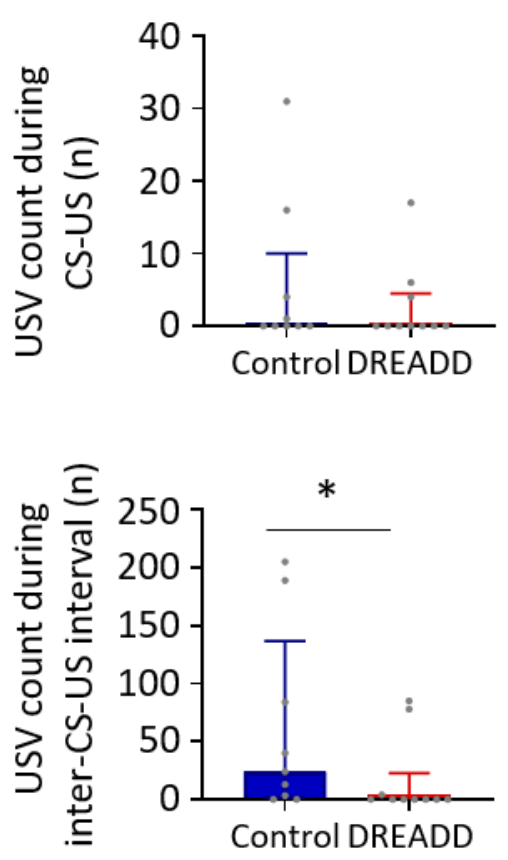

Figure 5. MCN-vPAG pathway inhibition and effect on behaviour during acquisition. A) The effect of CNO delivery into the VPAG on freezing behaviour measured during either presentation of the paired CS-US (upper plot), or the inter-CS-US interval (lower plot) during acquisition. Blue plot, control animals ( $n=9$ rats); red plot $\operatorname{DREADD}(\mathrm{hM} 4 \mathrm{D}(\mathrm{Gi}))$ animals, $\mathrm{n}=10$ rats); data points mean \pm SEM. B) The number of USVs recorded in control versus DREADD animals during CS-US presentation (upper panel) and during the inter-CS-US interval (lower panel); Bars show median \pm IQR. Mann Whitney, one tailed test, $* p<0.05$.

During extinction training, control and DREADD animals showed similar levels of freezing during presentation of the unreinforced CS+ (Fig. $6 A, E E p=0.645$, LE $p=0.362$, unpaired $t$ test,) and the interCS+ interval (Fig. 6B, EE $p=0.501, \mathrm{LE} \mathrm{p}=0.422$, unpaired t-test). Both groups also showed similar levels of extinction learning during CS+ (control, $p=0.0103$; DREADD, $p<0.0001$, paired $t$ test) and the inter$\mathrm{CS}+$ interval (control, $\mathrm{p}=0.0042$; DREADD, $\mathrm{p}<0.0001$, paired $\mathrm{t}$ tests). However, the rate of extinction during the CS+ was significantly slower in DREADD animals (Fig. 6C, $p=0.042$, unpaired t test), but not during the inter-CS+ interval (Fig. 6D, $p=0.229$, unpaired $t$ test). The latter finding suggests the effect is not a general disruption of the expression of freezing behaviour.

With regard to rearing behaviour no differences were found between control and DREADD animals both within early and late extinction (Fig. $6 \mathrm{E}, \mathrm{F}$ rearing; $\mathrm{EE} \mathrm{CS}+, \mathrm{p}=0.305$; LE inter-CS+ interval, $\mathrm{p}=0.903$; LE CS+, $p=0.273$; LE inter-CS+, $p=0.786$, unpaired $t$ test), and across all extinction training (Fig. $6 E, F E E$ vs LE; control CS+, $p=0.556$, control inter-CS+, $p=0.269$; DREADD, CS $+p=0.244$; DREADD, inter-CS+, $p=0.111$, paired t test,). Similarly, there was also no significant difference in the total number of USVs 
1 per animal in DREADD versus control groups across all extinction training (Fig. 6G,H CS+, p=0.844;

2 inter-CS+, $\mathrm{p}=0.849$, Mann Whitney tests).

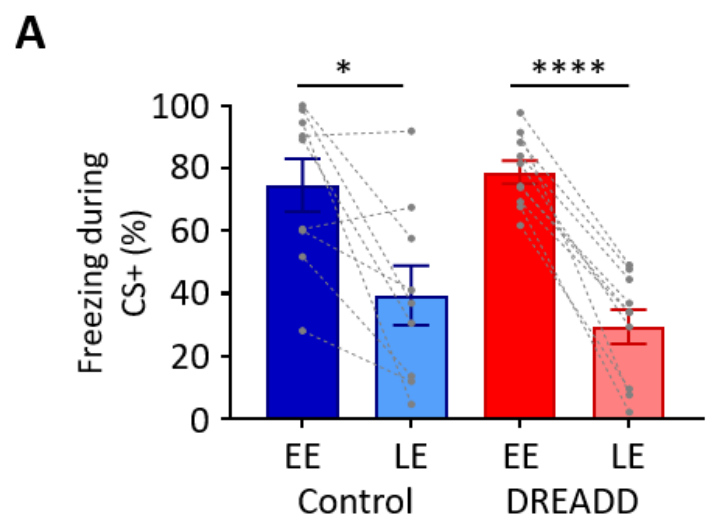

C

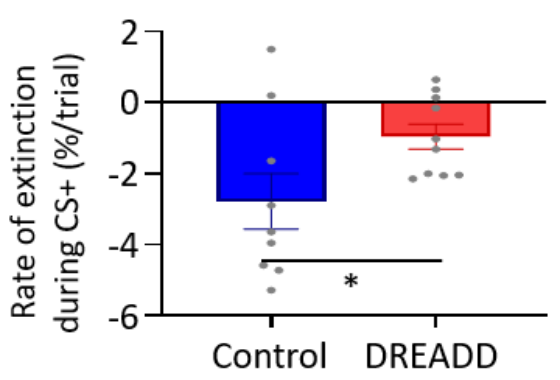

$\mathbf{E}$

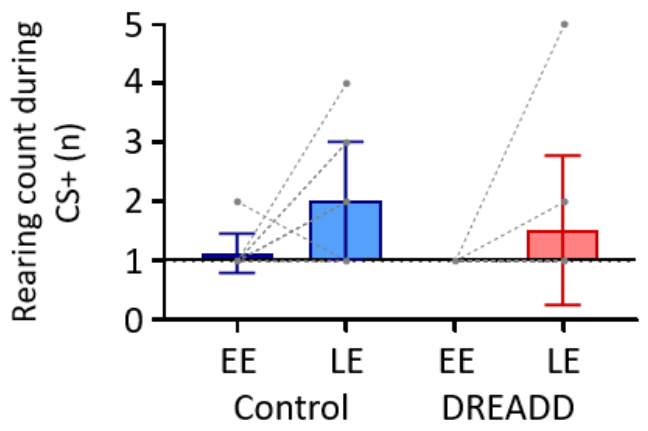

G

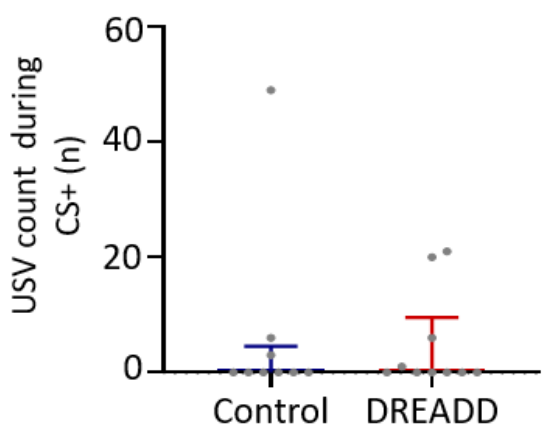

B

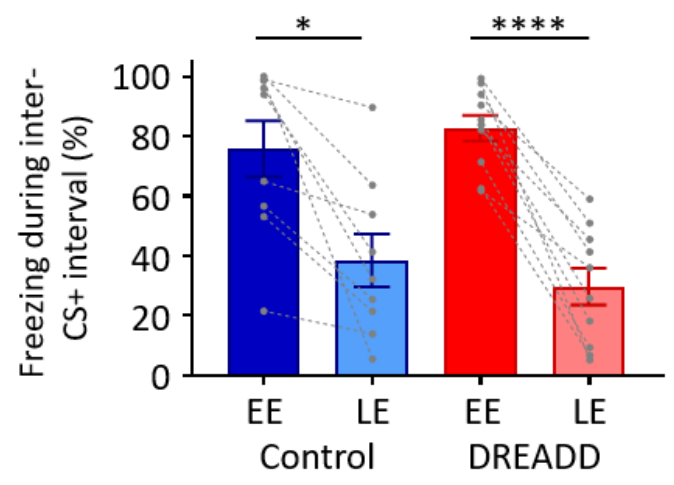

D

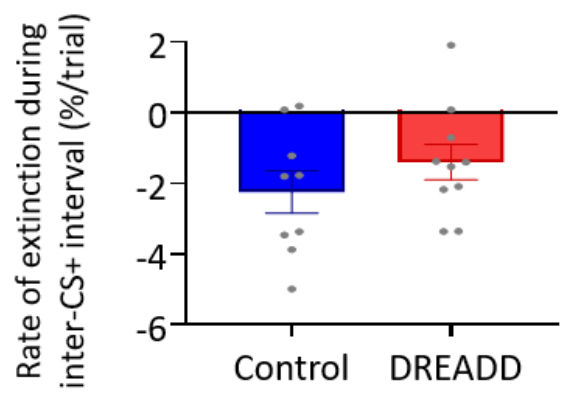

F

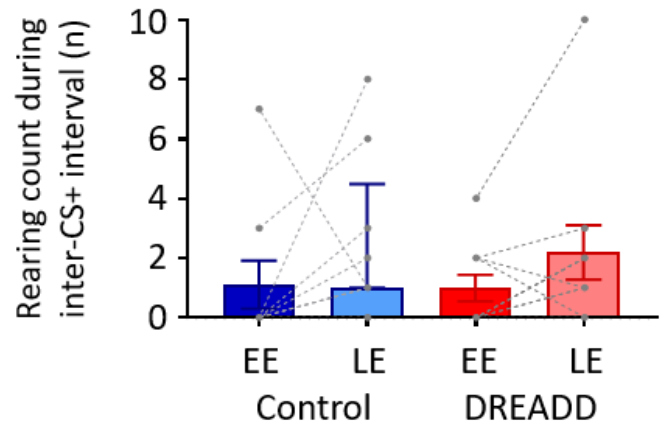

H

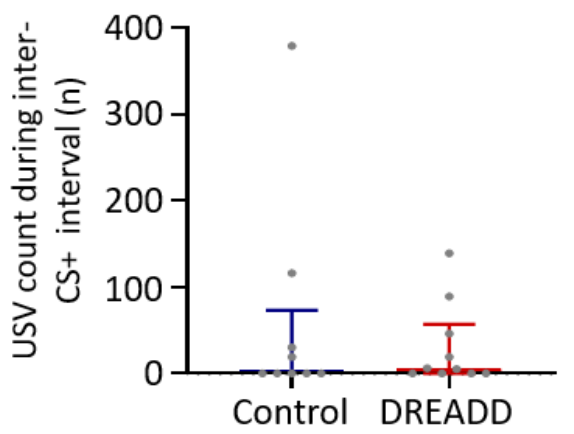

Figure 6. MCN-vPAG pathway inhibition effect on behaviour during extinction. A) The percentage of total time the CS+ was presented that animals displayed freezing behaviour during EE or LE in control ( $n=9$ rats) versus DREADD ( $\mathrm{hM} 4 \mathrm{D}(\mathrm{Gi}), \mathrm{n}=10$ rats). Individual data points connected with dashed lines show change in mean freezing time per animal over extinction training. Bars show group means \pm SEM. $* * * * p<0.0001,{ }^{*} p<0.05$. B) 
Same as A but percentage time freezing during the inter-CS+ interval. C) Rate of extinction as measured by the change in freezing percentage during presentation of the CS+ over the first 21 CS+ presentations during extinction training in control ( $n=9$ rats) versus DREADD ( $n=10$ rats) experiments. Individual data points show mean rate of change per animal. Bars show group means \pm SEM. Unpaired t-test $* p<0.05$. D) Same as $C$ but for inter-CS+ intervals. E) The total number of rears during presentation of the CS+ in extinction training in control ( $n=9$ rats) versus DREADD ( $n=10$ rats). Individual data points connected with dashed lines show change in mean number of rears per animal over extinction training. Bars show group median and IQR. F) Same as E but for interCS+ intervals. G) Total number of ultrasonic vocalisations (USVs) during presentation of the CS+ in extinction training in control ( $n=9$ rats) versus DREADD ( $n=10$ rats) experiments. Individual data points show mean count per animal. Bars show group median and IQR. H) Same as $\mathrm{G}$ but total number of USVs recorded during inter-CS+ interval.

\section{Control experiments for MCN-PAG inactivation}

To test for potential changes in motor and anxiety behaviour induced by inactivation of the MCN-PAG pathway, the same DREADD (and control) animals studied in the fear conditioning experiments ( $n=18$ rats) were also tested on the following tasks after $\mathrm{CNO}$ infusion: beam walking, open field and elevated plus maze. By comparison to controls, no change was found in performance on the beam (Supplementary Fig. $5 B$, time to traverse, $p=0.618$, unpaired t-test; number of foot slips, $p=0.500$, Mann-Whitney test); nor was there a difference in the distance travelled in an open field arena (Supplementary Fig. 5C, p=0.794, unpaired t-test), or the time spent in the centre versus the perimeter of the open field (Supplementary Fig. $5 D$, centre $p=0.887$; periphery $p=0.651$, unpaired t-test); or time spent in the open or closed arm of the elevated plus maze (Supplementary Fig. 5E, open arm, $p=0.635$; closed arm, $p=0.316$; unpaired t-test). Thus, no evidence was obtained to suggest that inactivation of the MCN-PAG causes a general deficit in motor behaviour or change in anxiety levels. However, insufficient inhibition of the MCN-PAG pathway cannot be ruled out. In all available animals (controls, $\mathrm{n}=8$; DREADD, $\mathrm{n}=9$ ) we therefore made an intraperitoneal injection of CNO in order to test for the effects of general inhibition of MCN. Consistent with the muscimol experiments, in every DREADD case this produced ataxia, although this was generally less severe and shorter lasting ( $\sim 1 \mathrm{~h})$ than was observed in the muscimol animals. For example, immediately after the infusion of muscimol, animals were unable to perform the beam walking task, while the DREADD animals were able to perform the task, although deficits were evident by comparison to baseline performance (Supplementary Fig. 5F, slower time to traverse, $p=0.006$, unpaired t-test; increased foot slips, $p=0.086$, Mann-Whitney test).

\section{Discussion.}

We have shown that VPAG neurons (type 1 units) encode temporally precise information about both the onset and offset of a fear conditioned auditory stimulus and that these two neuronal signals may be generated by independent mechanisms. This is because in VPAG during retrieval and extinction: i) some single units only respond to CS+ onset or only to CS+ offset; ii) unit onset and offset responses 
exhibited different characteristics during extinction training; iii) MCN inactivation disrupted the vPAG pattern of unit activity at CS+ offset but not onset; and (iv) the latency of the event related potential (ERP) at CS+ onset was significantly shorter than the ERP recorded at CS+ offset. Together, these findings imply different neural pathways generate onset and offset responses.

Importantly, vPAG units displayed little or no response to an auditory tone during habituation but displayed robust activity at tone onset and/or offset when the same tone was classically conditioned. This provides evidence that the responses were related to the associative conditioning rather than the sensory stimulus. Consistent with a recent study in mice (Frontera et al., 2020) the present results in rats found that inactivation of the MCN-vPAG pathway during acquisition reduces the subsequent rate of extinction of conditioned freezing behaviour during retrieval, and that MCN inactivation during consolidation has no detectable effect on fear conditioned freezing. However, the present behavioural results advance understanding beyond effects on freezing. Fear state involves a multiple pattern of defence-related responses, including (but not limited to) USVs and risk assessment behaviour such as rearing. We show that inactivation of the MCN-vPAG pathway during acquisition significantly increases the expression of fear-related USVs, and that MCN inactivation during consolidation significantly increases rearing during subsequent extinction training. Taken all together this raises the possibility that the MCN regulates different aspects of fear behaviour depending on the stage of conditioning (acquisition, retrieval, or extinction).

In regard to our electrophysiological findings, similar responses to CS+ onset in VPAG have been reported previously by ourselves (Watson et al., 2016) and others (Ozawa et al., 2017), but to our knowledge no equivalent response precisely time locked to CS+ offset has been described. This may be due to differences in the characteristics of the auditory cue used for conditioning (e.g., duration, intensity and rise-fall time of the tone: Takahashi et al., 2004; Qin et al., 2007; Scholl et al., 2010; Harris et al., 2017; Sołyga et al., 2019), but also, because detection of such responses depends on the temporal resolution used for the analysis. For example, Watson et al. (2016) used, as is the case in many other electrophysiological studies, a $1 \mathrm{sec}$ bin width to visualize the patterns of vPAG activity during CS+ presentation. Because offset responses are brief, temporally precise events, we found that a 40 ms bin width was needed to reliably capture them.

Fear conditioned CS+ onset responses in VPAG are thought to be generated as a result of preceding activity in the amygdala (Tovote et al., 2016;) and may encode multiple aspects of fear processing, including maintenance of fear memory after extinction (Watson et al., 2016), prediction error coding (Ozawa et al. 2017), the transmission of aversive teaching signals to the amygdala (Johansen et al., 2010) and threat probability (Wright and McDannald, 2019). vPAG activity has also been correlated to 
bioRxiv preprint doi: https://doi.org/10.1101/2021.02.17.431584; this version posted February 17, 2021. The copyright holder for this preprint (which was not certified by peer review) is the author/funder, who has granted bioRxiv a license to display the preprint in perpetuity. It is made available under aCC-BY 4.0 International license.

the onset of freezing behaviour (Tovote et al., 2016; Watson et al., 2016) indicating that there may be several distinct neuronal populations in the PAG that are responsive to conditioned stimulus onset. Our results for CS+ onset responses are consistent with Watson et al. (2016), as we show the phasic increase in neural activity was dependent on associative conditioning, and type 1 onset responses were generally similar in early and late extinction (ie were extinction resistant) and therefore may contribute to the persistence of fear memory after extinction.

In terms of previous reports of CS+ offset responses in VPAG, Wright and McDannald (2019) identified a distinct population of units in an auditory fear discrimination task they termed ramping units. These units were related to threat probability and also to fear output as determined by freezing behaviour. Ramping units progressively increase activity over the duration of the auditory cue presentation, reaching a peak around sound offset and ramping down in activity thereafter. However, this pattern differs markedly from our type 1 offset units whose phasic activity was precisely coupled to CS+ offset. Indeed, we found no evidence of ramping type activity in our sample of vPAG units. This is perhaps unsurprising because the experimental paradigm of Wright and McDannald (2019) differed from ours in a number of important ways. In particular, they used a trace fear conditioning protocol where the aversive footshock was paired in each session with the auditory cue with a varying probability of occurrence. The closest comparison with our results is between our acquisition sessions and their trials when the probability of a footshock was $100 \%$ (their Fig. 1). We observed an increase in activity immediately after the footshock and observed a progressive reduction in activity that resembled the change in firing after peak in their ramping units (our Fig. 1C).

An unanswered question concerns the origin of CS+ offset responses in vPAG. Our ERP data are consistent with offset responses being generated by synaptic input to the PAG, and we estimate that they occur approximately $29 \mathrm{~ms}$ after end of the CS+ tone. Such a delay provides ample opportunity for many possible pathways to generate them. Detecting the offset (and onset) of sensory events is a fundamental requirement of sensory processing by the CNS. Given the importance in the present experiments of the auditory system in the initial processing of the tone signals used for fear conditioning, one option is a route from the auditory cortex to the PAG. Both onset and offset responses have been reported in the auditory cortex elicited by tones and other sounds in a range of species (Qin et al., 2007; Tian et al., 2013; Liu et al., 2019). Also, changes in activity in the auditory association cortex during fear conditioning have been shown to precede the expected time of the unconditioned stimulus (Quirk et al., 1997). In principle, such activity occurs sufficiently in advance of our CS+ offset responses to be driving them. However, pathways from the auditory system to the PAG target its dorsolateral and lateral sectors and are associated with sound driven flight behaviour (e.g. Wang et al., 2019), so presumably some other pathway is responsible. For example, the medial 
prefrontal cortex and the bed nucleus of stria terminalis have extensive projections to the PAG (Holstege et al., 1985; An et al., 1998), and after fear conditioning both show sustained changes in activity during presentation of the CS+ (Haufler et al., 2013; Gilmartin et al., 2014).

A further important question relates to what information CS+ offset responses encode. Learning theory proposes that Pavlovian fear conditioning is instructed by an error signal that encodes the difference between actual and expected intensity of the US (Rescorla \& Wagner, 1972; Mcnally et al., 2011). The VPAG is generally considered to provide the teaching signal that encodes aversive prediction error to regulate synaptic plasticity in the amygdala and prefrontal cortex during fear extinction learning (Johansen et al., 2010; Mcnally et al., 2011; Roy et al., 2014; Walker et al., 2019; Frontera et al., 2020). According to the Rescorla-Wagner learning model this teaching signal is modulated by expectation of the US - during retrieval of a fear conditioned association the unreinforced CS+ produces a negative prediction error signal because the US has not occurred as expected. The reliability of this prediction is reduced with successive presentations of the unreinforced CS+. If fear extinction is instructed by this error signal, then neurons encoding prediction errors would be expected to progressively decrease their CS+ induced firing rate upon repeated omission of the expected US (McNally et al., 2011). In the present study the gradual reduction in CS+ type 1 offset responses in VPAG during extinction training are entirely consistent with this proposition. However, in our trace conditioning experiment the failure of CS+ offset responses to follow the timing of the expected US would seem to argue against this, although the $1 \mathrm{sec}$ time interval we used may have been too short for rats to discriminate. Another possibility is the CS+ offset response is signalling saliency of the tone, but this can also be thought of as a component of generating prediction error. Clearly, further studies are required, but the current findings open new avenues for investigating the role of VPAG in encoding fear memory.

We also show that inactivation of the cerebellar output nucleus MCN disrupts but does not abolish CS+ type 1 offset responses in vPAG. This is line with MCN modulating vPAG activity (Vaaga et al., 2020), but advances understanding by raising the possibility that the vermal compartment of the cerebellum is involved in the timing but not the origin of CS+ offset signals in vPAG. Consistent with this idea is the finding that in mice, phasic optogenetic stimulation of the MCN-VPAG pathway during CS+ offset significantly enhanced extinction learning, while tonic activation using chemogenetics had the opposite effect, suggesting that the temporal pattern of activation of VPAG neurons by MCN determines the effect on extinction learning (Frontera et al., 2020).

More generally, a role of $\mathrm{MCN}$ in temporal patterning is in good agreement with the timing hypothesis of cerebellar function (Ivry, 1997; Cheron et al., 2016; D'Angelo, 2018). This hypothesis proposes that 
the cerebellum not only regulates the timing of movement to enable coordinated behaviour and motor learning, but that this timing regulation extends to other functions of the CNS, including perceptual tasks that require the precise timing of salient events (Spencer et al., 2013). The present study extends this concept to the encoding of fear memory by vPAG. Our findings indicate that the cerebellum is important for the regulation of fear memory processes at multiple timescales: at the millisecond timescale to control CS+ offset timing within VPAG, and at longer timescales (hours/days) to regulate the rate of fear extinction and timing of expression of multiple fear-related behaviours. We provide evidence that MCN is not only involved in fear conditioned freezing behaviour but that it is also involved in the expression of USVs during acquisition and rearing behaviour during retrieval. It is tempting to speculate that MCN regulation of VPAG encoding of CS+ offset underlies some if not all of these behavioural effects, but this remains to be determined.

Cerebellar manipulations can affect emission of USVs (Fujita et al., 2008, 2012; Umeda et al., 2010; Fujita-Jimbo et al., 2014). Taken together with our USV results this raises the possibility that the MCNVPAG pathway regulates the emission of USVs at a time when danger is greatest, perhaps as a warning signal to conspecifics. Similarly, reduced rearing behaviour during early extinction may be a survival strategy to minimise detection by a predator at time when the probability of a threat is greatest. It is noteworthy that MCN receives its cerebellar cortical input from the cerebellar vermis and lesions of the pyramis (vermal lobule VIII) in rats result in deficits in innate and conditioned freezing behaviour but also a concomitant increase in risk assessment behaviours including rearing (Koutsikou et al., 2014). This suggests that expression of survival behaviours is regulated by the cerebellum in a context dependent manner. Our results also suggest that activity in the MCN-vPAG pathway during acquisition regulates the subsequent rate of fear extinction. This is consistent with a previous study in mice (Frontera et al., 2020) and suggests this is a function that is conserved across species. Outbred strains of rats have been shown to demonstrate different behavioural phenotypes during fear extinction ( $\mathrm{Ji}$ et al., 2018) where a proportion of animals show faster rates of extinction than others. Interruption of MCN-vPAG interaction during acquisition or early consolidation may therefore contribute to an anxiety-like behavioural phenotype, with wider implications for possible neural mechanisms that underly psychiatric disorders such as PTSD.

In summary, MCN regulates precise temporal encoding of fear memory within VPAG and also regulates the expression of different survival behaviours depending on the phase of Pavlovian fear conditioning. During early extinction MCN output supresses rearing, while a direct pathway to the VPAG appears to be important in eliciting fear related USVs during acquisition, and the rate of expression of freezing 
during early extinction. The cerebellum through its interactions with the survival network might therefore be coordinating the most appropriate behavioural response at the most appropriate time.

\section{Methods}

Animals: All animal procedures were performed in accordance with the UK Animals (Scientific Procedures) Act of 1986 and were approved by the University of Bristol Animal Welfare and Ethical Review Body. A total of 34 adult male Sprague Dawley rats (280-400g; Harlan Laboratories) were used in this study. They were housed under normal environmental conditions in a normal $12 \mathrm{~h}$ dark/light cycle and provided with food and water ad libitum. Animals were single housed after surgery to prevent damage to implants.

\section{Surgical procedures for chronic implants:}

Rats were anaesthetised initially with gaseous isoflurane, followed by intraperitoneal injections with ketamine and medetomidine $(5 \mathrm{mg} / 100 \mathrm{~g}$ of Narketan 10 and Domitor, Vetoquinol). Each animal was mounted in a stereotaxic frame with atraumatic ear bars and surgery was performed under aseptic conditions. Depth of anaesthesia was monitored regularly by testing corneal and paw withdrawal reflexes with supplementary doses of ketamine/medetomidine given as required. A midline scalp incision was made, and craniotomies were performed above the cerebellum and/or the PAG as required in each line of experiment. At the end of every surgery, the rat was administered the analgesic Metacam (Boehringer Ingelheim, $1 \mathrm{mg} / \mathrm{Kg}$ ) and the medetomidine antidote Atipamezole (Antisedan, Vetoquinol $0.1 \mathrm{mg}$ I.P.). Tetrodes, cannula and/or viral injections were carried out as described below depending on the experiment. Animals were handled for 1 week prior to surgery and during recovery before any behavioural paradigms or electrophysiological recordings.

\section{Electrophysiological recording ( $n=15$ rats):}

1. Dual microdrive experiments ( $n=7$ rats). Two in-house built microdrives, designed to slot closely next to each other, were positioned over craniotomies to allow tetrodes to be independently advanced into the right MCN (11.4 mm caudal from bregma, $1 \mathrm{~mm}$ lateral from midline, depth of $4 \mathrm{~mm}$ ) and contralateral vPAG (7.5 mm caudal from bregma, $1 \mathrm{~mm}$ lateral from midline, depth $4.8 \mathrm{~mm}$ ). The microdrives were attached to the skull with screws and dental acrylic cement. Each microdrive contained 3-4 tetrodes for local field potential (LFP) and single unit recording (0.0008-inch Tungsten wire 99.95\% CS $500 \mathrm{HML}$, insulated with VG Bond, $20 \mu \mathrm{m}$ inner diameter, impedance 100-400 K $\Omega$ after gold plating; California Fine Wire). 2. Single microdrive experiments ( $n=8$ rats). These implants were 
the same as described above except only one microdrive was implanted to record single units from the VPAG, and infusion cannulae were implanted bilaterally $(n=8)$ to target the MCN (details below).

Anatomical pathway tracing ( $n=7$ rats):

To anterogradely map direct connections between MCN and VIPAG, $100 \mathrm{nl}$ of an adeno associated viral (AAV) vector expressing tdTomato under the CAG promoter was injected unilaterally into the MCN (11.4 $\mathrm{mm}$ caudal from bregma, $1 \mathrm{~mm}$ lateral from midline, depth of $4.5 \mathrm{~mm}$ ). pAAV-CAG-tdTomato (codon diversified) was a gift from Edward Boyden (Addgene viral prep \# 59462-AAV1; http://n2t.net/addgene:59462; RRID:Addgene 59462). Injections of the AAV were made following previously published methods (Hirschberg et al., 2017). In brief, the glass micropipette) was connected to a $25 \mu \mathrm{l}$ syringe (Hamilton, Bonaduz, Switzerland) via tubing filled with mineral oil, and was then backfilled with virus using a syringe driver (AL-1000, World Precision Instruments). To monitor progress of the injection, movement of the oil-vector capillary interface was monitored. Injections were made at $200 \mathrm{nl} / \mathrm{min}$ and the pipette left in situ for $5 \mathrm{~min}$ prior to removal.

Inhibitory DREADD surgery ( $n=19$ rats):

For the inhibitory DREADD experiments two adeno associated viral (AAV) vectors were used: a control, pAAV-hSyn-EGFP (AAV5); and an inhibitory DREADD, pAAV-hSyn-hM4D(Gi)-mCherry (AAV5) (both gifts from Bryan Roth, Addgene viral prep \# 50465-AAV5 and \# 50475-AAV5). Using the same techniques as outlined above, animals were injected bilaterally into MCN with $350 \mathrm{nl}$ of either the control $(n=9)$ or the inhibitory DREADD $(n=10)$. In the same surgery bilateral cannulae (26-gauge guide cannula, PlasticsOne) were also chronically implanted with tips (33 gauge internal) located just above the vIPAG (7.5mm caudal from bregma, $0.8 \mathrm{~mm}$ lateral from midline, at a depth of $5 \mathrm{~mm}$ ).

\section{Behavioural protocols:}

Auditory cued fear conditioning ( $n=34$ rats). The delay conditioning paradigm was based on Watson et al (2016, Fig. 1A). On day 0 all animals underwent a session of habituation to the Skinner box (Med Associates Inc., St Albans, VT, USA) to act as a baseline for analysis prior to a session of acquisition (day 1). During acquisition, the conditioned stimulus (CS, $2 \mathrm{KHz}, 10 \mathrm{sec}$ tone) was paired $7 \mathrm{x}$ with an unconditioned stimulus (US, $0.5 \mathrm{~s}$ footshock, $0.75 \mathrm{~mA}$ ) delivered at the end of the tone, except in one animal where the timing of the US was delayed $1 \mathrm{sec}$ after the CS. This was followed by a session of extinction training (day 2). During extinction, 5 blocks of 7 tone presentations (trials) were repeated. The first two blocks (trials 1-14) were defined as early extinction (EE), when the animal was exhibiting high levels of freezing, while the last two blocks (trials 21-35) were defined as late extinction (LE), when the animals were exhibiting low levels of freezing. 
Balance beam ( $n=18$ rats). This test was used to assess general motor coordination and balance (Luong et al., 2011). Animals were trained for 3 consecutive days to cross 6 times a $160 \mathrm{~cm}$ long beam that ended on an enclosed safety platform. On each day, the beam was progressively thinner in width $(6 \mathrm{~cm}, 4 \mathrm{~cm}$ and $2 \mathrm{~cm})$. The $2 \mathrm{~cm}$ beam was then used for the test day. Baseline performance was recorded and then $\mathrm{CNO}$ (Clozapine $\mathrm{N}$-oxide) was given either i.p. or by intracranial infusion (for details see below) and after an interval of 15 minutes the animal was re-tested on the beam. Beam balance performance was manually scored using Solomon Coder software (C 2019 by András Péter), by scoring the time to cross the beam for each trial and the number of foot slips.

Open field ( $n=14$ rats). This test was used to assess both general motor behaviour and anxiety levels. Animals were exposed for the first time to the arena (round arena with $90 \mathrm{~cm}$ diameter, and hight of $51 \mathrm{~cm}$ ) on the test day. They were placed at the perimeter of the arena and were allowed to explore for 10 minutes. Exploratory behaviour was recorded for the whole session. DeepLabCut (Wei et al., 2018) was used to track the animal behaviour in the video, the tracking output was then used to calculate total distance covered and time spent in two equivalent areas of the arena, a central and a peripheral one.

Elevated plus maze ( $n=18$ rats). This test was used as an additional assessment of anxiety (Pellow et al., 1985). Animals were placed in a plus shaped maze, $1 \mathrm{~m}$ above the floor, with 2 open and 2 closed arms (10 cm wide and $50 \mathrm{~cm}$ length) and allowed to explore the maze for 5 minutes. Elevated plus maze performance was manually scored using Solomon encoder software (@ 2019 by András Péter) to calculate total time spent in open versus closed arms and the number of entries into each arm.

\section{Data acquisition and analysis:}

Electrophysiological recording. Multisite electrophysiological data were recorded using a Blackrock Microsystems (Utah, USA) data capture system synchronised with OptiTrak software. Neural data were sampled at $30 \mathrm{kHz}$ and band pass filtered online between $300 \mathrm{~Hz}-6 \mathrm{kHz}$.

Ultrasonic vocalisation recordings. USVs emitted at $22 \mathrm{kHz}$ were recorded using an ANL-940-1 Ultrasonic Microphone and Amplifier (Med Associates, Inc.) connected to the Blackrock Microsystems (Utah, USA). Although USVs were recorded as an aliased signal (the maximum sampling rate of the recording system was $30 \mathrm{kHz}$, while the optimal sampling rate was $44 \mathrm{kHz}$ ) we were able to reliably identify all USV events. For analysis, USVs were visualised using Spike7 software (Cambridge Electronic Design Limited) and individual USV emissions manually identified and the total number during each recording session was counted. 
Auditory cued fear conditioning. Video recording of animal behaviour during the fear conditioning paradigm was captured with an OptiTrak camera and software, allowing synchronisation with neural data. Fear-related freezing behaviour was manually scored using Solomon Coder software (@ 2019 by András Péter). Freezing behaviour was identified as periods of time longer than $2 \mathrm{~s}$ in which the animal had an absence of movement (except those associated with respiration and eye movements, Blanchard \& Blanchard, 1969) while typically maintaining a crouching position. Percentages of time spent freezing were calculated for each trial during CS+ presentations and during inter-CS+ intervals. To evaluate the extinction rate of freezing behaviour, the slope of the intercept of freezing $\%$ for all trials was calculated. Rearing activity was counted as events in which the animal was standing upright on its rear limbs. The number of rearing events during CS+ presentations and inter-CS+ intervals was counted.

Control behavioural tests: Beam balance, open field and elevated plus maze tests were recorded via standard webcams linked to OBS (Open Broadcaster Software; (C) 2012-2020).

Inactivation experiments $(n=4)$. In 4 animals muscimol (Sigma Aldrich, $0.3 \mathrm{ul}$, at a rate of $0.3 \mathrm{ul} / \mathrm{min}$ ) (or saline; $n=4$ animals) was infused via indwelling cannulae at a rate of $0.3 \mathrm{ul} / \mathrm{min}$ to target $\mathrm{MCN}$. The infusion was made immediately after acquisition (i.e., during early consolidation). Extinction training was carried out 48 hours after the infusion.

\section{Inhibitory DREADD experiments:}

A total of 19 animals were randomly assigned to either a saline control $(n=9)$ or a DREADD experimental group $(n=10)$ and coded so the experimenter was blinded. Unblinding occurred once all procedures and analysis were completed. Six weeks after viral transfection (see above), animals were tested in the following behavioural paradigms: auditory cued fear conditioning, beam balance, open field and elevated plus maze. One animal was excluded from the study after the fear conditioning test because of poor health; a further 3 animals were excluded from the open field analysis because of technical problems with the video recording. In every animal a volume of $500 \mathrm{nl}$ of $\mathrm{CNO}$ ( $3 \mu \mathrm{M}$, Tocris) was infused at a rate of $0.5 \mu \mathrm{l} / \mathrm{min}$ to target the PAG, 15 minutes prior to each behavioural test (infusion pump Harvard Apparatus, PHD 2000 Infusion). During fear conditioning, since the effect of CNO is estimated to last 60-90 mins (Stachniak et al., 2014; Jendryka et al., 2019) this meant the pathway was likely to be inhibited during both acquisition and also a period of early consolidation. In one experiment, to verify functional activity of $h M 4 D(G i)$ receptors in the CNS following MCN transfection with inhibitory DREADD, CNO was injected i.p. ( $2.5 \mathrm{mg} / \mathrm{Kg}) 20$ minutes before the beam balance test. 
2 At experimental end points all animals were deeply anaesthetized (Euthatal, $200 \mathrm{mg} \mathrm{ml}^{-1}$, Merial

3 Animal Health) and terminated by transcardial perfusion (4\% paraformaldehyde in $0.1 \mathrm{M}$ phosphate buffer) and the brains extracted. After post-fixation, the brains were cryoprotected in $30 \%$ sucrose solution. The cerebellum was cut sagittally and the midbrain including the PAG cut coronally, into sections of 40 or $60 \mu \mathrm{m}$ thickness, respectively. To aid verification of electrode and cannula brain loci, sections were stained with cresyl violet. For visualisation of viral expression immunohistochemistry anti-mCherry (1:2000, Anti-mCherry Polyclonal Antibody host rabbit, BioVision, with 5\% normal horse serum (NHS); 1:1000, Alexafluor 594 donkey anti Rabbit IgG, Molecular probes) was performed. No signal amplification was required for the eGFP controls. Sections were visualised on an Axioskop 2 Plus microscope (Zeiss) and photomicrographs captured using AxioVision software, or with a widefield microscope (Leica DMI6000, with Leica DFC365FX camera and Leica LASX live cell imaging workstation software).

Neural data analysis:

Spike sorting. Offline spike sorting was carried out using MClust software in Matlab. Clustering was classified as single unit if $L$ ratio $<0.35$, ID $>15$ and $<1 \%$ of interspike intervals was $>2 \mathrm{~ms}$ (See Schmitzer-Torbert et al., 2005). The firing rate of individual units was taken from MClust and verified using NeuroExplorer.

Data analysis of single units:

For habituation and acquisition recording sessions peri event time histograms (PETHs, 40ms time bins) of the activity of individual units were created in NeuroExplorer with tone onset and offset as time zero. During acquisition, the footshock caused electrical interference so it was not possible to analyse unit activity during the $0.5 \mathrm{sec}$ period of stimulus delivery. The following analysis was performed on MATLAB. In all experimental groups PETHs of unit activity to the unreinforced conditioned tone (CS+) during extinction training were constructed for individual units over all trials (1-35) and also separately for early extinction (trials 1-14) and late extinction trials (21-35). PETHs were z-score normalised to a 5 second baseline recording of unit activity before tone onset and data grouped to show the average of all unit responses and separated into different unit response types. A significant response was defined as 1 or more consecutive 40 ms time bins where the $z$-score was \pm 2 SD from baseline mean. The peak response was measured as maximal value found within the first $25 \times 40 \mathrm{~ms}$ bins after tone onset or offset. The area of the response was calculated as the trapezoidal numerical integration of the first $25 \times 40 \mathrm{~ms}$ bins after tone onset or offset. 
Data analysis of Local field potential. Local field potential (LFP) data were averaged in relation to tone onset and offset using MATLAB ( $n=14$ trials per mean of each animal). The tetrode recording site yielding the largest mean peak to trough response was identified in each animal and used to calculate group average data of peak amplitude of LFP response recorded in the PAG and cerebellum.

Statistical analysis. Statistical analysis and graphs were performed with GraphPad Prism 9. Data was shown as mean \pm S.E.M., except for rearing behaviour and USVs that were shown as median $\pm I Q R$. Paired t-tests or Wilcoxon test (for USVs and rearing behaviour) were used for within group comparison, while unpaired t-tests or Mann Whitney test (for USVs and rearing behaviour) and ANOVA were used to compare groups. Differences were considered significant at $p<0.05$.

Acknowledgments: We gratefully acknowledge Ms Rachel Bissett for her help with histological processing and the Wolfson Bioimaging Facility for their support and assistance. We would like to thank Bryan Roth and Edward Boyden for supplying the viral vectors used in this work. This work was supported by BBSRC grant BB/MO19616/1 and a Wellcome Trust PhD studentship 203775/Z/16/Z.

Competing interests: The authors declare that no competing interests exist.

\section{References:}

Albert, Dempesy, \& Sorenson. (1985). Anterior cerebellar vermal stimulation: Effect on behavior and basal forebrain neurochemistry in rat. Biological Psychiatry, 20(12), 1267-1276. https://doi.org/10.1016/0006-3223(85)90111-8

An, Bandler, Öngür, \& Price. (1998). Prefrontal cortical projections to longitudinal columns in the midbrain periaqueductal gray in macaque monkeys. Journal of Comparative Neurology, 401(4), 455-479. https://doi.org/10.1002/(SICI)1096-9861(19981130)401:4<455::AID-CNE3>3.0.CO;2-6

Apps, \& Strata. (2015). Neuronal circuits for fear and anxiety - the missing link. Nature Reviews Neuroscience, 16(SEPTEMBER), 4-6. https://doi.org/10.1038/nrn4028

Asdourian, \& Frerichs. (1970). Some effects of cerebellar stimulation. Psychonomic Science, 18(5), 261-262. https://doi.org/10.3758/BF03331817

Bares, Lungu, Liu, Waechter, Gomez, \& Ashe. (2007). Impaired predictive motor timing in patients with cerebellar disorders. Experimental Brain Research, 180(2), 355-365. https://doi.org/10.1007/s00221-007-0857-8

Blanchard, \& Blanchard. (1969). Crouching as an index of fear. Journal of Comparative and Physiological Psychology, 67(3), 370-375. https://doi.org/10.1037/h0026779

Bremner, Staib, Kaloupek, Southwick, Soufer, \& Charney. (1999). Neural correlates of exposure to traumatic pictures and sound in Vietnam combat veterans with and without posttraumatic stress disorder: A positron emission tomography study. Biological Psychiatry, 45(7), 806-816. https://doi.org/10.1016/S0006-3223(98)00297-2

Cacciola, Bertino, Basile, Di Mauro, Calamuneri, Chillemi, Duca, Bruschetta, Flace, Favaloro, Calabrò, Anastasi, \& Milardi. (2019). Mapping the structural connectivity between the periaqueductal gray and the cerebellum in humans. Brain Structure and Function, 224(6), 2153-2165. 
https://doi.org/10.1007/s00429-019-01893-x

Cheron, Márquez-Ruiz, \& Dan. (2016). Oscillations, Timing, Plasticity, and Learning in the Cerebellum. In Cerebellum (Vol. 15, Issue 2, pp. 122-138). Springer New York LLC. https://doi.org/10.1007/s12311-015-0665-9

Clelland, Choi, Romberg, Jr, Fragniere, \& Tyers. (2010). NIH Public Access. 325(5937), 210-213. https://doi.org/10.1126/science.1173215.A

D’Angelo. (2018). Physiology of the cerebellum. In Handbook of Clinical Neurology (Vol. 154, pp. 85108). Elsevier B.V. https://doi.org/10.1016/B978-0-444-63956-1.00006-0

Frontera, Aissa, Sala, Mailhes-Hamon, Georgescu, Léna, \& Popa. (2020). Bidirectional control of fear memories by cerebellar neurons projecting to the ventrolateral periaqueductal grey. Nature Communications 2020 11:1, 11(1), 1-17. https://doi.org/10.1038/s41467-020-18953-0

Fujita-Jimbo, \& Momoi. (2014). Specific expression of FOXP2 in cerebellum improves ultrasonic vocalization in heterozygous but not in homozygous Foxp2 (R552H) knock-in pups. Neuroscience Letters, 566, 162-166. https://doi.org/10.1016/j.neulet.2014.02.062

Fujita, Tanabe, Imhof, Momoi, \& Momoi. (2012). Cadm1-Expressing synapses on Purkinje cell dendrites are involved in mouse ultrasonic vocalization activity. PLOS ONE, 7(1). https://doi.org/10.1371/journal.pone.0030151

Fujita, Tanabe, Shiota, Ueda, Suwa, Momoi, \& Momoi. (2008). Ultrasonic vocalization impairment of Foxp2 (R552H) knockin mice related to speech-language disorder and abnormality of Purkinje cells. Proceedings of the National Academy of Sciences of the United States of America, 105(8), 3117-3122. https://doi.org/10.1073/pnas.0712298105

Gilmartin, Balderston, \& Helmstetter. (2014). Prefrontal cortical regulation of fear learning. Trends in Neurosciences, 37(8), 455-464. https://doi.org/10.1016/j.tins.2014.05.004

Harris, Golder, \& Likhtik. (2017). Multisite Electrophysiology Recordings in Mice to Study CrossRegional Communication During Anxiety. In Current Protocols in Neuroscience (pp. 8.40.18.40.21). John Wiley \& Sons, Inc. https://doi.org/10.1002/cpns.32

Haufler, Nagy, \& Pare. (2013). Neuronal correlates of fear conditioning in the bed nucleus of the stria terminalis. Learning and Memory, 20(11), 633-641. https://doi.org/10.1101/Im.031799.113

Heinricher, Cheng, \& Fields. (1987). Evidence for two classes of nociceptive modulating neurons in the periaqueductal gray. Journal of Neuroscience, 7(1), 271-278. https://doi.org/10.1523/jneurosci.07-01-00271.1987

Herry, Ferraguti, Singewald, Letzkus, Ehrlich, \& Lüthi. (2010). Neuronal circuits of fear extinction. In European Journal of Neuroscience (Vol. 31, Issue 4, pp. 599-612). Eur J Neurosci. https://doi.org/10.1111/j.1460-9568.2010.07101.x

Hirschberg, Li, Randall, Kremer, \& Pickering. (2017). Functional dichotomy in spinal- vs prefrontalprojecting locus coeruleus modules splits descending noradrenergic analgesia from ascending aversion and anxiety in rats. ELife, 6, e29808. https://doi.org/10.7554/eLife.29808

Holstege, Meiners, \& Tan. (1985). Projections of the bed nucleus of the stria terminalis to the mesencephalon, pons, and medulla oblongata in the cat. Experimental Brain Research, 58(2), 379-391. https://doi.org/10.1007/BF00235319

Ivry. (1997). Cerebellar timing systems. International Review of Neurobiology, 41, 555-573. https://doi.org/10.1016/s0074-7742(08)60370-0 
Jendryka, Palchaudhuri, Ursu, van der Veen, Liss, Kätzel, Nissen, \& Pekcec. (2019). Pharmacokinetic and pharmacodynamic actions of clozapine-N-oxide, clozapine, and compound 21 in DREADDbased chemogenetics in mice. Scientific Reports, 9(1). https://doi.org/10.1038/s41598-01941088-2

Ji, Yakhnitsa, Kiritoshi, Presto, \& Neugebauer. (2018). Fear extinction learning ability predicts neuropathic pain behaviors and amygdala activity in male rats. Molecular Pain, 14. https://doi.org/10.1177/1744806918804441

Johansen, Tarpley, LeDoux, \& Blair. (2010). Neural substrates for expectation-modulated fear learning in the amygdala and periaqueductal gray. Nature Neuroscience, 13(8), 979-986. https://doi.org/10.1038/nn.2594

Johansson, Hesslow, \& Medina. (2016). Mechanisms for motor timing in the cerebellar cortex. In Current Opinion in Behavioral Sciences (Vol. 8, pp. 53-59). Elsevier Ltd. https://doi.org/10.1016/j.cobeha.2016.01.013

Kim, Horovitz, Pellman, Tan, Li, Richter-Levin, \& Kim. (2013). Dorsal periaqueductal gray-amygdala pathway conveys both innate and learned fear responses in rats. Proceedings of the National Academy of Sciences of the United States of America, 110(36), 14795-14800. https://doi.org/10.1073/pnas.1310845110

Koch, Oliveri, Torriero, Salerno, Gerfo, \& Caltagirone. (2007). Repetitive TMS of cerebellum interferes with millisecond time processing. Experimental Brain Research, 179(2), 291-299. https://doi.org/10.1007/s00221-006-0791-1

Koutsikou, Crook, Earl, Leith, Watson, Lumb, \& Apps. (2014). Neural substrates underlying fearevoked freezing: the periaqueductal grey-cerebellar link. The Journal of Physiology, 592(10), 2197-2213. https://doi.org/10.1113/jphysiol.2013.268714

Liu, Whiteway, Sheikhattar, Butts, Babadi, \& Kanold Correspondence. (2019). Parallel Processing of Sound Dynamics across Mouse Auditory Cortex via Spatially Patterned Thalamic Inputs and Distinct Areal Intracortical Circuits. CellReports, 27, 872-885.e7. https://doi.org/10.1016/j.celrep.2019.03.069

Luong, Carlisle, Southwell, \& Patterson. (2011). Assessment of motor balance and coordination in mice using the balance beam. Journal of Visualized Experiments : JoVE, 49. https://doi.org/10.3791/2376

McNally, Johansen, \& Blair. (2011). Placing prediction into the fear circuit. Trends in Neurosciences, 34(6), 283-292. https://doi.org/10.1016/j.tins.2011.03.005

Milad, \& Quirk. (2012). Fear extinction as a model for translational neuroscience: Ten years of progress. In Annual Review of Psychology (Vol. 63, pp. 129-151). NIH Public Access. https://doi.org/10.1146/annurev.psych.121208.131631

Nisimaru, Mittal, Shirai, Sooksawate, Anandaraj, Hashikawa, Nagao, Arata, Sakurai, Yamamoto, \& Ito. (2013). Orexin-neuromodulated cerebellar circuit controls redistribution of arterial blood flows for defense behavior in rabbits. Proceedings of the National Academy of Sciences of the United States of America, 110(35), 14124-14131. https://doi.org/10.1073/pnas.1312804110

Ouda, Jílek, \& Syka. (2016). Expression of c-Fos in rat auditory and limbic systems following 22-kHz calls. Behavioural Brain Research, 308, 196-204. https://doi.org/10.1016/j.bbr.2016.04.030

Ozawa, Ycu, Kumar, Yeh, Ahmed, Koivumaa, \& Johansen. (2017). A feedback neural circuit for calibrating aversive memory strength. Nature Neuroscience, 20(1), 90-97. https://doi.org/10.1038/nn.4439 
Pellow, Chopin, File, \& Briley. (1985). Validation of open : closed arm entries in an elevated plusmaze as a measure of anxiety in the rat. Journal of Neuroscience Methods, 14(3), 149-167. https://doi.org/10.1016/0165-0270(85)90031-7

Qin, Chimoto, Sakai, Wang, \& Sato. (2007). Comparison Between Offset and Onset Responses of Primary Auditory Cortex - Neurons in Awake Cats. Journal of Neurophysiology, 97(5), 34213431. https://doi.org/10.1152/jn.00184.2007

Quirk, Armony, \& LeDoux. (1997). Fear conditioning enhances different temporal components of tone-evoked spike trains in auditory cortex and lateral amygdala. Neuron, 19(3), 613-624. https://doi.org/10.1016/S0896-6273(00)80375-X

Rescorla, R. Wagner. (1972). A theory of Pavlovian conditioning: Variations in the effectiveness of reinforcement and nonreinforcement. Classical Conditioning II: Current Research and Theory, January 1972, 64-99.

Roy, Shohamy, Daw, Jepma, Wimmer, \& Wager. (2014). Representation of aversive prediction errors in the human periaqueductal gray. Nature Neuroscience, 17(11), 1607-1612. https://doi.org/10.1038/nn.3832

Sacchetti, B., Scelfo, \& Strata. (2009). Cerebellum and emotional behavior. Neuroscience, 162(3), 756-762. https://doi.org/10.1016/j.neuroscience.2009.01.064

Sacchetti, Benedetto, Scelfo, \& Strata. (2005). The Cerebellum: Synaptic Changes and Fear Conditioning. The Neuroscientist, 11(3), 217-227. https://doi.org/10.1177/1073858405276428

Sanders, Klein, Mayer, Heym, \& Handwerker. (1980). Differential effects of noxious and non-noxious input on neurones according to location in ventral periaqueductal grey or dorsal raphe nucleus. Brain Research, 186(1), 83-97. https://doi.org/10.1016/0006-8993(80)90257-7

Sandner, Schmitt, \& Karli. (1987). Mapping of jumping, rearing, squealing and switch-off behaviors elicited by periaqueductal gray stimulation in the rat. Physiology \& Behavior, 39(3), 333-339. https://doi.org/10.1016/0031-9384(87)90231-9

Schmitzer-Torbert, Jackson, Henze, Harris, \& Redish. (2005). Quantitative measures of cluster quality for use in extracellular recordings. Neuroscience, 131(1), 1-11. https://doi.org/10.1016/j.neuroscience.2004.09.066

Scholl, Gao, \& Wehr. (2010). Nonoverlapping Sets of Synapses Drive On Responses and Off Responses in Auditory Cortex. Neuron, 65(3), 412-421. https://doi.org/10.1016/j.neuron.2010.01.020

Sharma, Sinha, Mathur, \& Nayar. (1999). Neuronal responses of periaqueductal gray to peripheral noxious stimulation. Indian Journal of Physiology and Pharmacology, 43(4), 449-457. https://europepmc.org/article/med/10776460

Sołyga, \& Barkat. (2019). Distinct processing of tone offset in two primary auditory cortices. Scientific Reports, 9(1), 1-12. https://doi.org/10.1038/s41598-019-45952-z

Spencer, \& Ivry. (2013). Cerebellum and timing. In Handbook of the Cerebellum and Cerebellar Disorders (pp. 1201-1220). Springer Netherlands. https://doi.org/10.1007/978-94-007-13338 82

Stachniak, Ghosh, \& Sternson. (2014). Chemogenetic Synaptic Silencing of Neural Circuits Localizes a Hypothalamus $\rightarrow$ Midbrain Pathway for Feeding Behavior. Neuron, 82(4), 797-808. https://doi.org/10.1016/J.NEURON.2014.04.008

Strick, Dum, \& Fiez. (2009). Cerebellum and Nonmotor Function. Annual Review of Neuroscience, 
bioRxiv preprint doi: https://doi.org/10.1101/2021.02.17.431584; this version posted February 17,2021 . The copyright holder for this preprint (which was not certified by peer review) is the author/funder, who has granted bioRxiv a license to display the preprint in perpetuity. It is made available under aCC-BY 4.0 International license.

32(1), 413-434. https://doi.org/10.1146/annurev.neuro.31.060407.125606

Supple, \& Leaton. (1990). Cerebellar vermis: essential for classically conditioned bradycardia in the rat. Brain Research, 509(1), 17-23. https://doi.org/10.1016/0006-8993(90)90303-S

Supple, Leaton, \& Fanselow. (1987). Effects of cerebellar vermal lesions on species-specific fear responses, neophobia, and taste-aversion learning in rats. Physiology \& Behavior, 39(5), 579586. https://doi.org/10.1016/0031-9384(87)90156-9

Takahashi, Nakao, \& Kaga. (2004). Cortical mapping of auditory-evoked offset responses in rats. NeuroReport, 15(10), 1565-1569. https://doi.org/10.1097/01.wnr.0000134848.63755.5c

Teune, van der Burg, van der Moer, Voogd, \& Ruigrok. (2000). Topography of cerebellar nuclear projections to the brain stem in the rat. In Progress in brain research (Vol. 124, pp. 141-172). https://doi.org/10.1016/S0079-6123(00)24014-4

Tian, Kuśmierek, \& Rauschecker. (2013). Analogues of simple and complex cells in rhesus monkey auditory cortex. Proceedings of the National Academy of Sciences of the United States of America, 110(19), 7892-7897. https://doi.org/10.1073/pnas.1221062110

Toledo, Lang, Doengi, Morrison, Stein, \& Baader. (2019). Merlin modulates process outgrowth and synaptogenesis in the cerebellum. Brain Structure and Function, 224(6), 2121-2142. https://doi.org/10.1007/s00429-019-01897-7

Tovote, Esposito, Botta, Chaudun, Fadok, Markovic, Wolff, Ramakrishnan, Fenno, Deisseroth, Herry, Arber, \& Lüthi. (2016). Midbrain circuits for defensive behaviour. Nature, 534(7606), 206-212. https://doi.org/10.1038/nature17996

Tovote, Fadok, \& Lüthi. (2015). Neuronal circuits for fear and anxiety. Nature Reviews Neuroscience, 16(6), 317-331. https://doi.org/10.1038/nrn3945

Umeda, Takashima, Nakagawa, Maekawa, Ikegami, Yoshikawa, Kobayashi, Okanoya, Inokuchi, \& Osumi. (2010). Evaluation of pax6 mutant rat as a model for autism. PLOS ONE, 5(12). https://doi.org/10.1371/journal.pone.0015500

Vaaga, Brown, \& Raman. (2020). Cerebellar modulation of synaptic input to freezing-related neurons in the periaqueductal gray. ELife, 9. https://doi.org/10.7554/eLife.54302

Vianna, Graeff, Brandão, \& Landeira-Fernandez. (2001). Defensive freezing evoked by electrical stimulation of the periaqueductal gray: comparison between dorsolateral and ventrolateral regions. Neuroreport, 12(18), 4109-4112. http://www.ncbi.nlm.nih.gov/pubmed/11742247

Walker, P., \& Carrive. (2003). Role of ventrolateral periaqueductal gray neurons in the behavioral and cardiovascular responses to contextual conditioned fear and poststress recovery. Neuroscience, 116(3), 897-912. https://doi.org/10.1016/S0306-4522(02)00744-3

Walker, R. A., Wright, Jhou, \& McDannald. (2019). The ventrolateral periaqueductal grey updates fear via positive prediction error. European Journal of Neuroscience, 51(3), 866-880. https://doi.org/10.1111/ejn.14536

Wang, Chen, Xu, Sun, Chen, Zhao, Luo, Liu, Guo, Xie, Zhong, Bai, Tian, Mao, Ye, Tao, Li, Farzinpour, Li, ... Zhang. (2019). Direct auditory cortical input to the lateral periaqueductal gray controls sound-driven defensive behavior. PLOS Biology, 17(8), e3000417. https://doi.org/10.1371/journal.pbio.3000417

Watson, Cerminara, Lumb, \& Apps. (2016). Neural Correlates of Fear in the Periaqueductal Gray. The Journal of Neuroscience, 36(50), 12707-12719. https://doi.org/10.1523/JNEUROSCl.110016.2016 
bioRxiv preprint doi: https://doi org/10.1101/2021.02 17.431584 this version posted February 17, 2021. The copyright holder for this preprint (which was not certified by peer review) is the author/funder, who has granted bioRxiv a license to display the preprint in perpetuity. It is made available under aCC-BY 4.0 International license.

1

2

3

4

5

6

7

8

9

10

Wei, \& Kording. (2018). Behavioral tracking gets real. Nature Neuroscience, 1. https://doi.org/10.1038/s41593-018-0215-0

Whiteside, \& Snider. (1953). Relation of cerebellum to upper brain stem. Journal of Neurophysiology, 16(4), 397-413. https://doi.org/10.1152/jn.1953.16.4.397

Wright, \& McDannald. (2019). Ventrolateral periaqueductal gray neurons prioritize threat probability over fear output. ELife, 8. https://doi.org/10.7554/eLife.45013

$\mathrm{Xu}, \mathrm{Liu}$, Ashe, \& Bushara. (2006). Role of the olivo-cerebellar system in timing. Journal of Neuroscience, 26(22), 5990-5995. https://doi.org/10.1523/JNEUROSCI.0038-06.2006 
A
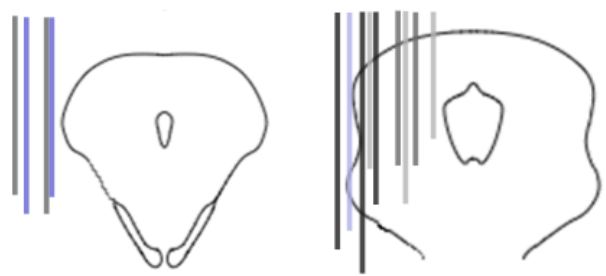

B
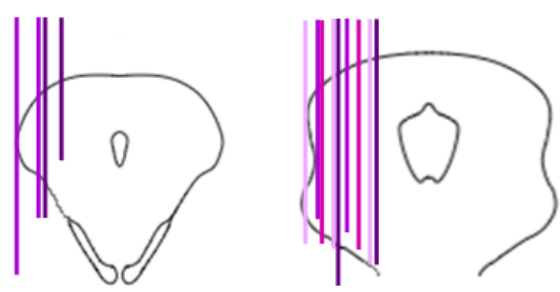

C
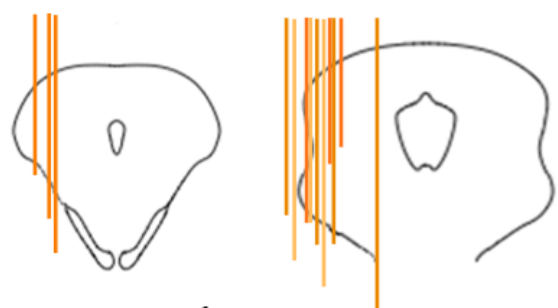

$1 \mathrm{~mm}$

$-6.5 A P$
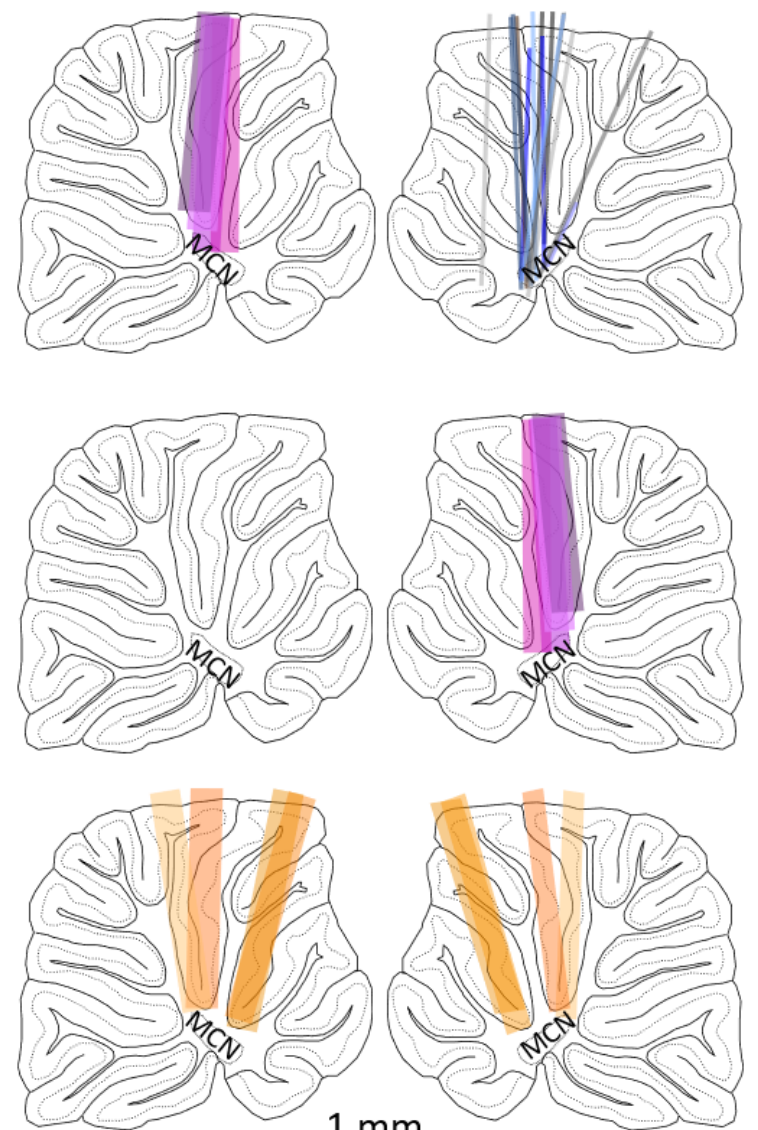

$1 \mathrm{~mm}$

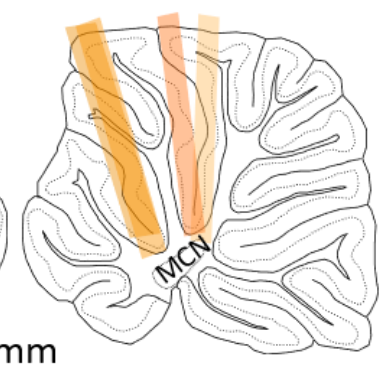

$-1 \mathrm{ML}$

$+1 \mathrm{ML}$

Supplementary Figure 1. Histological verification of recording electrodes (microdrive implants) and infusion cannulae location. A) Control animals with dual microdrive implant ( $n=6$ rats, each animal tracks colour coded blue to black); reconstruction of tetrode tracks in the VPAG (left, coronal sections at AP levels -6.5 and -7.5 ) and contralateral MCN (right, sagittal sections $\pm 1 \mathrm{~mm}$ from midline). B) Animals with single microdrive implant and cannulae used for saline infusions ( $n=4$ rats, each animal tracks colour coded with purple shade); reconstruction of tetrode tracks in the VPAG (left) and cannulae placement in the left and right cerebellar MCN (right). C) Same as $B$ but cannulae used for infusions of muscimol ( $n=4$ rats, each animal tracks colour coded with orange shade). 


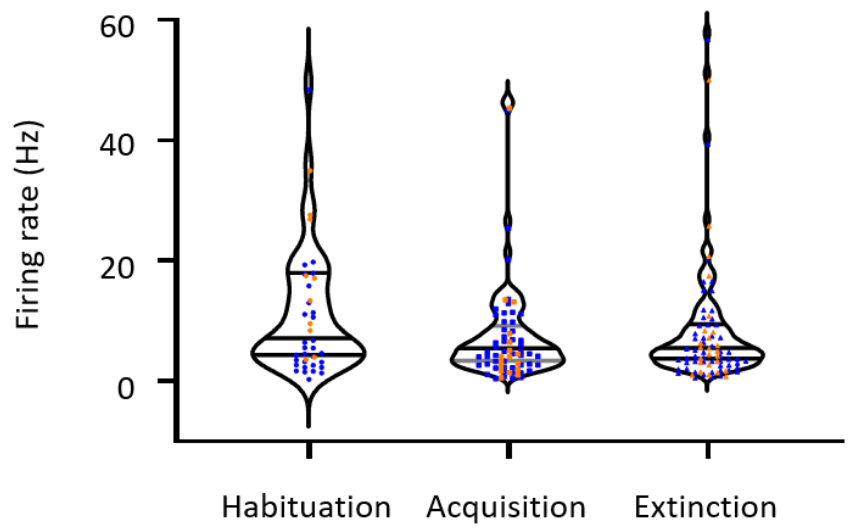

2 Supplementary Figure 2. Firing rate properties. Violin plots showing distribution of baseline firing rates of all available single units recorded in vPAG during habituation ( $n=30$ control units, $n=10$ muscimol units), acquisition ( $n=50$ control units, $n=17$ muscimol units) or extinction ( $n=55$ control units, $n=25$ muscimol units). Horizontal lines show median and IQR firing rate per phase of conditioning. Blue dots, control data; orange dots, muscimol data.

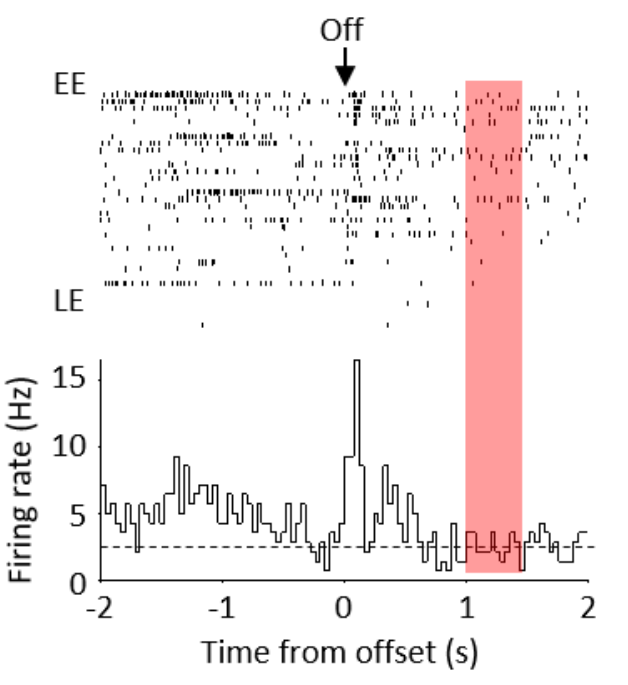

Supplementary Figure 3. vPAG offset responses during auditory cued fear trace conditioning. Example single unit in VPAG recorded during extinction following acquisition where a 1 second trace was introduced between CS offset and the US. Raster plot and peri-event time histogram showing activity relative to CS+ offset (arrow in raster and time zero in PETH). Onset and duration of the US during acquisition is shown by red shaded area; PETH 40ms bins. 
bioRxiv preprint doi: https://doi org/10.1101/2021.02 17.431584; this version posted February 17, 2021. The copyright holder for this preprint (which was not certified by peer review) is the author/funder, who has granted bioRxiv a license to display the preprint in perpetuity. It is made available under aCC-BY 4.0 International license.

A

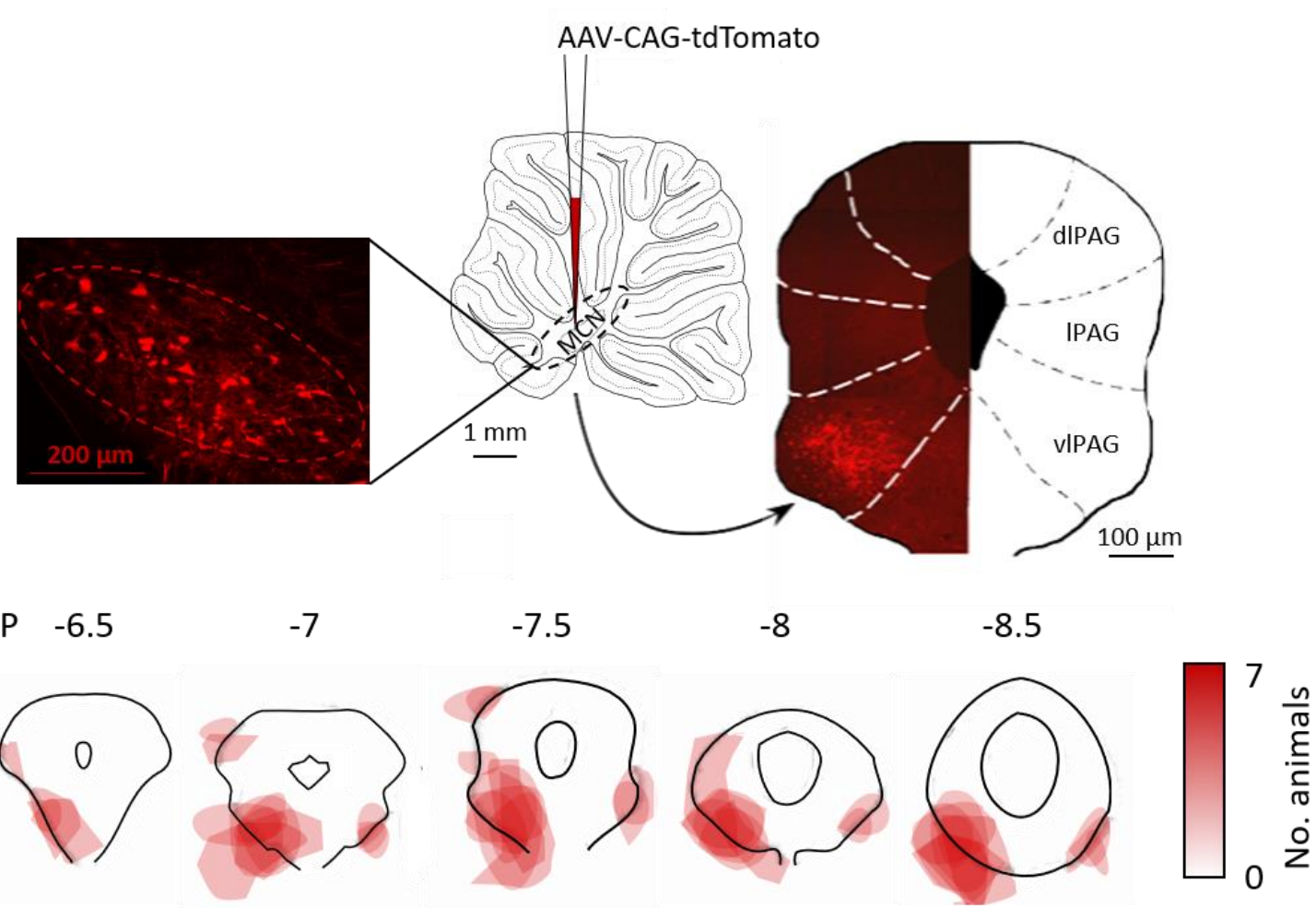

Supplementary Figure 4. Anatomical mapping of the MCN-PAG pathway. A) Schematic showing on a sagittal section of the cerebellum injection into the MCN of an anterograde tracer (AAV-CAG-tdTomato viral vector) to label direct projections to the PAG. Coronal section shows representative pattern of terminal labelling in the contralateral PAG in one animal (dIPAG, IPAG, vIPAG; dorsolateral, lateral and ventrolateral periaqueductal grey). B) Coronal sections of the PAG at different anterioposterior (AP) levels showing the pattern of terminal labelling in all available animals ( $n=7$ rats). The darker the shading the larger the number of animals with terminal labelling in that area. 
A

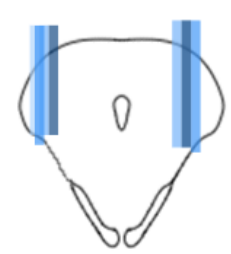

-6.5 AP

B

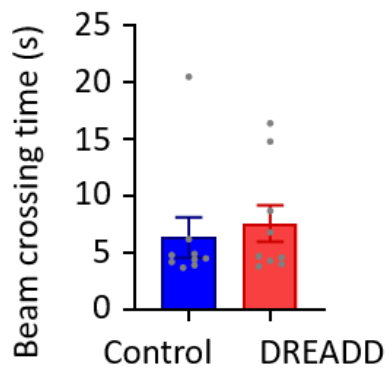

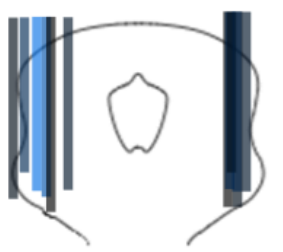

$-7.5 \mathrm{AP}$

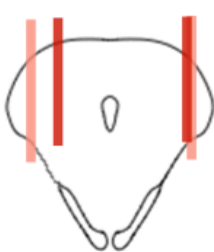

$-6.5 \mathrm{AP}$

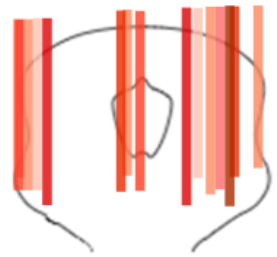

$-7.5 \mathrm{AP}$
C

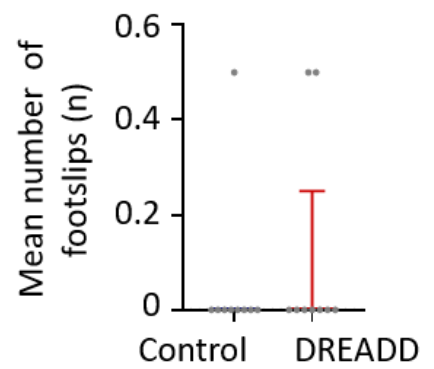

D

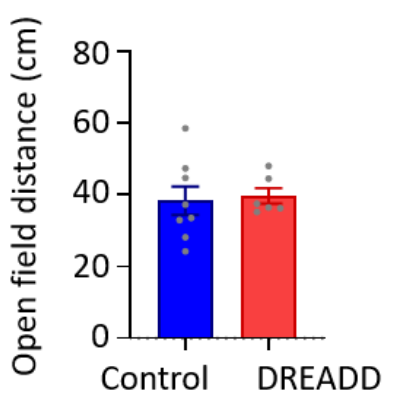

E

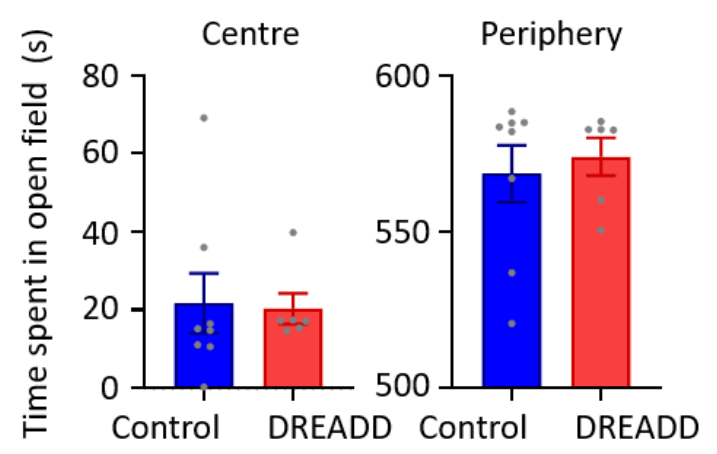

F

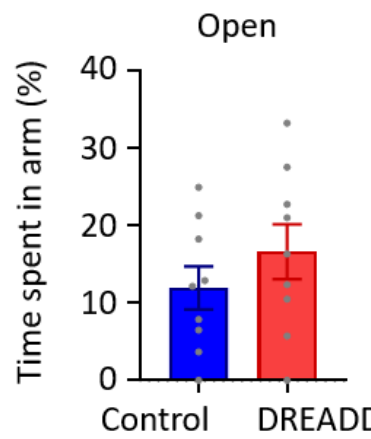

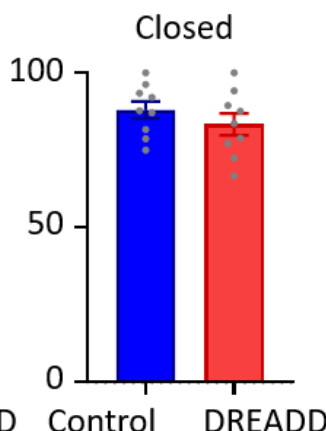

1

G

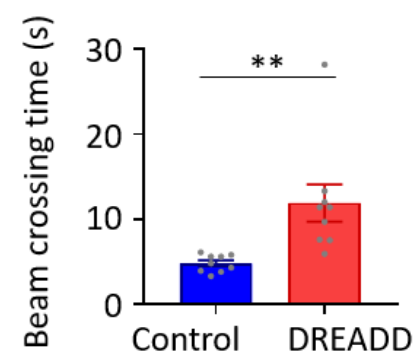

H

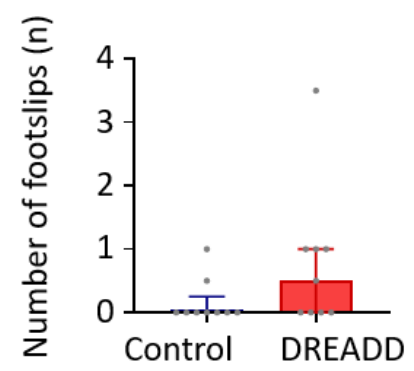

Supplementary figure 5. Effect of DREADDs on general motor and affective behaviour. A) Histological identification of cannulae placement in PAG in control ( $n=9$ rats, tracks colour coded blue to black for different animals) and DREADD animals ( $n=10$ rats, tracks colour coded orange to red for different animals). B) Beam walking crossing times in control ( $n=9$ rats) and DREADD ( $n=9$ rats) experiments after infusion of CNO into vPAG. Individual data points show mean crossing time per animal. Bars show mean \pm SEM C) Same as $B$ but mean number of footslips in control ( $n=9$ rats) versus DREADD ( $n=9$ rats) experiments. Individual data points show mean number of footslips per animal. Bars show median \& IQR. D) The total distance moved in an open field arena in control ( $n=8$ rats) versus DREADD ( $n=6$ rats) experiments. Individual data points show total distance per animal. Bars show mean \pm SEM. E) Time spent in the centre versus the periphery of the open field arena for control versus DREADD animals. Individual data points show total time per animal. Bars show mean \pm SEM. F) Percentage of the total time spent in the open arm (left) and in the closed arm (right) of an elevated plus maze for control ( $n=9$ rats) versus DREADD ( $n=9$ rats) experiments. Individual data points show percentage time per animal. Bars show mean \pm SEM. G) Effect of intraperitoneal injection of CNO on the total crossing time in the beam walking task for DREADD ( $n=9$ rats) versus control ( $n=9$ rats) experiments. Individual data points show 
bioRxiv preprint doi: https://doi.org/10.1101/2021.02.17.431584; this version posted February 17, 2021. The copyright holder for this preprint (which was not certified by peer review) is the author/funder, who has granted bioRxiv a license to display the preprint in perpetuity. It is made available under aCC-BY 4.0 International license.

time per animal. Bars show mean \pm SEM. Unpaired t-test $* *, p<0.001$. H) Same as $G$ but for the number of footslips. Bars show mean \pm SEM. 